\title{
An optimizing reduced PLSMFE formulation for non-stationary conduction-convection problems*
}

\section{Zhen-Dong Luo}

School of Science, Beijing Jiaotong University, Beijing 100044, China; e-mail: zhdluo@bjtu.edu.cn

\section{Jing Chen}

College of Science, China Agricultural University, Beijing 100083, China; e-mail: jing_quchen@163.com

\section{I.M. Navon}

School of Computational Science and Department of Mathematics, Florida State University, Dirac Sci. Lib. Bldg., \#483, Tallahassee, FL 32306-4120, USA; e-mail: navon@scs.fsu.edu

Received

Summary. In this work, proper orthogonal decomposition (POD) is combined with Petrov-Galerkin least squares mixed finite element (PLSMFE) method to derive an optimizing reduced PLSMFE formulation for non-stationary conduction-convection problems. Error estimates between the optimizing reduced PLSMFE solutions based on POD and classical MFE solutions are presented. The optimizing reduced PLSMFE formulation can circumvent the constraint of Babuška-Brezzi (BB) condition so that the combination of finite element subspaces can be chosen freely and allow optimal order error estimates to be obtained. Numerical simulation examples have shown that the errors between the optimizing reduced PLSMFE solutions and the classical MFE solutions are consistent with theoretical results. Moreover, they have also shown the feasibility and efficiency of POD method.

Mathematics Subject Classifications (2000): 65N30, 35Q10

\section{Introduction}

Let $\Omega \subset \mathbb{R}^{2}$ be a bounded, connected, and polygonal domain. Consider the following the non-stationary conduction-convection problems, where the coupled equations govern viscous incompressible flow and heat transfer processes for an incompressible fluid are Boussinesq approximations to non-stationary Navier-Stokes equations.

Problem (I) Find $u=\left(u_{1}, u_{2}\right), p$ and $T$ such that for $t_{N}>0$,

$$
\begin{cases}u_{t}-\nu \Delta u+(u \cdot \nabla) u+\nabla p=\gamma j T & (x, y, t) \in \Omega \times\left(0, t_{N}\right), \\ \nabla \cdot u=0 & (x, y, t) \in \Omega \times\left(0, t_{N}\right), \\ T_{t}-\gamma^{-1} \Delta T+u \cdot \nabla T=0 & (x, y, t) \in \Omega \times\left(0, t_{N}\right), \\ u(x, y, t)=0, \quad T(x, y, t)=\varphi(x, y, t) & (x, y, t) \in \partial \Omega \times\left(0, t_{N}\right), \\ u(x, y, 0)=0, T(x, y, 0)=\psi(x, y) & (x, y) \in \Omega,\end{cases}
$$

Correspondence to: Jing Chen

* This work was supported in part by the National Science Foundation of China (NSF10471100 and NSF40437017) and Beijing Jiaotong University Science Technology Foundation 
where $u=\left(u_{1}, u_{2}\right)$ represents the velocity vector, $p$ the pressure, $T$ the temperature, $\nu=R e^{-1}$, Re the Reynolds number, $\gamma>0$ is the Groshoff number, $j=(0,1)$ the unit vector, and $\varphi(x, y, t)$ and $\psi(x, y)$ are the given functions. $t_{N}$ is the final time. For the sake of convenience and without lost generality, we may as well suppose that $\varphi(x, y, t)=0$ in the following theoretical analysis.

The non-stationary conduction-convection problems (I) constitute an important system of equations in atmospheric dynamics and a compelling dissipative nonlinear system of equations. Since this system of equations does not only contain velocity vector field and the pressure field, but also contains the temperature field ${ }^{[1]}$, finding the numerical solution of Problem (I) is not easy. Though mixed finite element (MFE) method is one of the important approaches for solving the non-stationary conduction-convection problems, the fully discrete system of MFE solutions for the non-stationary conduction-convection problems has many degrees of freedom and it is an important convergence stability condition that the Babuška-Brezzi (BB) inequality ${ }^{[2-4]}$ holds for the combination of finite element subspaces. Thus, an important problem is how to circumvent the constraint of the BB inequality and to simplify the computational load and save time-consuming calculations and resource demands in the actual computational process in a way that guarantees a sufficiently accurate numerical solution. In order to circumvent the constraint of the BB inequality in studies of MFE methods for Stokes equation and Navier-Stokes Equations, stabilized finite element methods ${ }^{[5-8]}$ have been developed, motivated by streamline diffusion $(\mathrm{SD})$ methods $^{[9-10]}$. A stabilized SD method ${ }^{[11]}$ for the stationary Navier-Stokes Equations is also proposed. Some Petrov-Galerkin least squares methods for the stationary Navier-Stokes equations and non stationary conduction convection problems which residuals are added to were developed ${ }^{[12-13]}$. Proper orthogonal decomposition (POD) is a technique offering adequate approximation for representing fluid flow with reduced number of degrees of freedom, i.e., with lower dimensional models so as to alleviate the computational load and provide CPU and memory requirements savingsi.POD has be successfully used in different fields including signal analysis and pattern recognition ${ }^{[14-15]}$, fluid dynamics and coherent structures ${ }^{[16-21]}$, as well as optimal flow control problems ${ }^{[22-24]}$. More recently, some reduced order finite difference models and MFE formulations and error estimates for the upper tropical pacific ocean model based on POD were presented ${ }^{[25-28]}$, and an optimizing finite difference scheme based on POD for the non-stationary conduction-convection problems was established ${ }^{[29]}$. However, to the best of our knowledge, there are no published results to address the issue where POD is used to reduce the PLSMFE formulation for the non-stationary conduction-convection problems and provide error estimates between classical PLSMFE solutions and reduced PLSMFE solutions. Therefore, in this paper, we combine PLSMFE methods with POD to deal with the non-stationary conduction-convection problems so that we insure not only the stabilization of solutions of fully discrete PLSMFE system, but we also simplify the computational load and save time-consuming calculations and resource demands in the actual computational process in a way that guarantees a sufficiently accurate numerical solution. We also derive error estimates between usual PLSMFE solutions and the solutions of optimizing reduced PLSMFE formulation based POD technique. Then we consider the results obtained from numerical simulations 
of cavity flows to show that the errors between POD solutions of optimizing reduced PLSMFE formulation and the usual PLSMFE solutions are consistent with theoretical results. Moreover, we also show the feasibility and efficiency of the POD method.

Though Kunisch and Volkwein have presented some Galerkin POD methods for parabolic problems ${ }^{[30]}$ and a general equation in fluid dynamics ${ }^{[31]}$, our method is different from their approaches, whose methods consist of Galerkin projection approaches where the original variables are substituted for linear combination of POD basis and the error estimates of the velocity field therein are only derived, while their POD basis is generated with the solution of the physical system at all time instances. In particular, in our present method the velocity field is only approximated $^{[31]}$, while velocity and pressure fields are all simultaneously approximated . Although the singular value decomposition approach combined with POD technique is used to treat the Burgers equation in [32] and the cavity flow problem in [33], the error estimates have not completely been derived, in particular, an optimizing reduced formulation of PLSMFE for the non-stationary conduction-convection problems has not yet been derived. Therefore, our method improves upon existing methods and POD basis is only generated along with the numerical solution at a subset of time instances which are useful and of interest for us.

The present paper is organized as follows. In Section 2 we derive usual PLSMFE methods for the non-stationary conduction-convection problems and generate snapshots from transient solutions computed from the equation system derived by usual PLSMFE methods. In section 3, the optimal orthogonal bases are reconstructed from the elements of the snapshots with POD and an optimizing reduced PLSMFE formulation is developed with lower dimensional number based on POD for the nonlinear non-stationary conduction-convection problems . In section 4, error estimates between usual PLSMFE solutions and POD solutions of optimizing reduced PLSMFE formulation are derived. In section 5, some numerical examples are presented illustrating that the errors between optimizing the PLSMFE approximate solutions and the usual PLSMFE solutions are consistent with previously obtained theoretical results, thus validating both the feasibility and efficiency of POD method. Section 6 provides the main conclusions and future tentative ideas.

\section{Usual PLSMFE approximation for the non-stationary conduction- convection problems and snapshots generate}

The Sobolev spaces along with their properties used in this context are standard ${ }^{[34]}$. For example, for a bounded domain $\Omega$, we denote by $H^{m}(\Omega)(m \geq 0)$ and $L^{2}(\Omega)=H^{0}(\Omega)$ the usual Sobolev spaces equipped with the semi-norm and the norm, respectively,

$$
|v|_{m, \Omega}=\left\{\sum_{|\alpha|=m} \int_{\Omega}\left|D^{\alpha} v\right|^{2} \mathrm{~d} x \mathrm{~d} y\right\}^{1 / 2} \text { and }\|v\|_{m, \Omega}=\left\{\sum_{i=0}^{m}|v|_{i, \Omega}^{2}\right\}^{1 / 2} \forall v \in H^{m}(\Omega)
$$

where $\alpha=\left(\alpha_{1}, \alpha_{2}\right), \alpha_{1}$ and $\alpha_{2}$ are two nonnegative integers, and $|\alpha|=\alpha_{1}+\alpha_{2}$. Especially, the 
subspace $H_{0}^{1}(\Omega)$ of $H^{1}(\Omega)$ is denoted by

$$
H_{0}^{1}(\Omega)=\left\{v \in H^{1}(\Omega) ;\left.u\right|_{\partial \Omega}=0\right\} .
$$

Note that $\|\cdot\|_{1}$ is equivalent to $|\cdot|_{1}$ in $H_{0}^{1}(\Omega)$. Let $L_{0}^{2}(\Omega)=\left\{q \in L^{2}(\Omega) ; \int_{\Omega} q \mathrm{~d} x \mathrm{~d} y=0\right\}$, which is the subspace of $L^{2}(\Omega)$.

$$
\begin{aligned}
& \text { Put } X=H_{0}^{1}(\Omega)^{2}, M=L_{0}(\Omega)=\left\{q \in L^{2}(\Omega) ; \int_{\Omega} q \mathrm{~d} x \mathrm{~d} y=0\right\}, W=H_{0}^{1}(\Omega), \text { and } \\
& a(u, v)=\nu \int_{\Omega} \nabla u \cdot \nabla v \mathrm{~d} x \mathrm{~d} y \quad \forall u, v \in X, \\
& b(q, v)=\int_{\Omega} q \operatorname{div} v \mathrm{~d} x \mathrm{~d} y \quad \forall v \in X, q \in M, \\
& a_{1}(u, v, w)=\frac{1}{2} \int_{\Omega} \sum_{i, j=1}^{2}\left[u_{i} \frac{\partial v_{j}}{\partial x_{i}} w_{j}-u_{i} \frac{\partial w_{j}}{\partial x_{i}} v_{j}\right] \mathrm{d} x \mathrm{~d} y \quad \forall u, v, w \in X, \\
& a_{2}(u, T, \phi)=\frac{1}{2} \int_{\Omega} \sum_{i=1}^{2}\left[u_{i} \frac{\partial T}{\partial x_{i}} \phi-u_{i} \frac{\partial \phi}{\partial x_{i}} T\right] \mathrm{d} x \mathrm{~d} y \quad \forall u \in X, \forall T, \phi \in W, \\
& D(T, \phi)=\gamma^{-1} \int_{\Omega} \nabla T \cdot \nabla \phi \mathrm{d} x \mathrm{~d} y \quad \forall T, \phi \in W .
\end{aligned}
$$

Let $N$ be the positive integer, denote the time step increment by $k=t_{N} / N$. Write $t_{n}=k n$ and $\left(u^{n}, p^{n}, T^{n}\right)$ denotes the semi-discrete approximation of $\left(u\left(x, y, t_{n}\right), p\left(x, y, t_{n}\right), T\left(x, y, t_{n}\right)\right)$. By introducing a finite difference approximation for time derivation of Problem (I), we obtain the following semi-discrete formulation at discrete times.

Problem (II) Find $\left(u^{n}, p^{n}\right) \in X \times M$ such that for $n=1,2, \cdots, N$,

$$
\left\{\begin{array}{l}
\left(u^{n}, v\right)+k a\left(u^{n}, v\right)+k a_{1}\left(u^{n}, u^{n}, v\right)-k b\left(p^{n}, v\right) \\
=k \gamma\left(j T^{n}, v\right)+\left(u^{n-1}, v\right) \quad \forall v \in X, \\
b\left(q, u^{n}\right)=0 \quad \forall q \in M, \\
\left(T^{n}, \phi\right)+k D\left(T^{n}, \phi\right)+k a_{2}\left(u^{n}, T^{n}, \phi\right)=\left(T^{n-1}, \phi\right) \quad \forall \phi \in W, \\
u^{0}=0, \quad T^{0}=\psi(x, y) \text { in } \Omega .
\end{array}\right.
$$

Using the theory of stationary conduction-convection problems may prove that Problem (II) has a unique solution, and has the following error estimate ${ }^{[1,35]}$.

THEOREM 2.1. If second derivatives $u_{t t}$ and $T_{t t}$ of the solution $(u, p, T)$ of Problem (I) are all bounded, then

$$
\begin{aligned}
& \left\|u\left(t_{n}\right)-u^{n}\right\|_{0}+(k \nu)^{\frac{1}{2}} \sum_{i=1}^{n}\left|u\left(t_{i}\right)-u^{i}\right|_{1}+\left\|T\left(t_{n}\right)-T^{n}\right\|_{0} \\
& +\left(k \gamma^{-1}\right)^{\frac{1}{2}} \sum_{i=1}^{n}\left|T\left(t_{i}\right)-T^{i}\right|_{1}+k^{\frac{1}{2}} \sum_{i=1}^{n}\left\|p\left(t_{i}\right)-p^{i}\right\|_{0} \leq C k
\end{aligned}
$$

where $\left(u\left(t_{n}\right), p\left(t_{n}\right), T\left(t_{n}\right)\right)$ is the value at $t_{n}=k n$ of the solution $(u(t), p(t), T(t))$ of Problem $(\mathrm{I}), C$ is a constant dependent on $(u(t), p(t), T(t))$ but independent of $k$. 
In order to find the numerical solution for Problem (II), it is necessary to discretize Problem (II). We introduce a MFE approximation for the spatial variable. Let $\left\{\Im_{h}\right\}$ be a uniformly regular family of triangulation of $\bar{\Omega}^{[35-36]}$, indexed by a parameter $h=\max _{K \in \Im_{h}}\left\{h_{K} ; h_{K}=\operatorname{diam}(K)\right\}$, i.e., there is a constant $C$, independent of $h$, such that $h \leq C h_{K}\left(\forall K \in \Im_{h}\right)$. We introduce the finite element subspaces $X_{h} \subset X, M_{h} \subset M$, and $W_{h} \subset W$ as follows

$$
\begin{aligned}
& X_{h}=\left\{v_{h} \in X \cap C^{0}(\bar{\Omega})^{2} ;\left.v_{h}\right|_{K} \in P_{\ell}(K)^{2} \quad \forall K \in \Im_{h}\right\}, \\
& M_{h}=\left\{q_{h} \in M \cap C^{0}(\bar{\Omega}) ;\left.q_{h}\right|_{K} \in P_{\kappa}(K) \quad \forall K \in \Im_{h}\right\}, \\
& W_{h}=\left\{\phi_{h} \in W \cap C^{0}(\bar{\Omega}) ;\left.\phi_{h}\right|_{K} \in P_{\iota}(K) \quad \forall K \in \Im_{h}\right\},
\end{aligned}
$$

where $P_{\ell}(K)$ is the space of piecewise polynomials of degree $\ell$ on $K, \ell \geq 1, \kappa \geq 1$, and $\iota \geq 1$ are three integers.

Let $\left(u_{h}^{n}, p_{h}^{n}, T_{h}^{n}\right) \in X_{h} \times M_{h} \times W_{h}$ be the PLSMFE approximation corresponding to $\left(u^{n}, p^{n}\right.$, $T^{n}$ ). Then, the fully discrete PLSMFE solution for the problem (II) may be written as:

Problem (III). Find $\left(u_{h}^{n}, p_{h}^{n}, T_{h}^{n}\right) \in X_{h} \times M_{h} \times W_{h}$ such that for $1 \leq n \leq N$,

$$
\begin{aligned}
& \left(u_{h}^{n}, v_{h}\right)+k a\left(u_{h}^{n}, v_{h}\right)+k a_{1}\left(u_{h}^{n}, u_{h}^{n}, v_{h}\right)-k b\left(p_{h}^{n}, v_{h}\right)+k b\left(q_{h}, u_{h}^{n}\right)+ \\
& \sum_{K \in \Im_{h}} \delta_{K}\left(u_{h}^{n}-k \nu \triangle u_{h}^{n}+k\left(u_{h}^{n} \cdot \nabla\right) u_{h}+k \nabla p_{h}^{n}, v_{h}-k \nu \triangle v_{h}+k\left(u_{h}^{n} \cdot \nabla\right) v_{h}+k \nabla q_{h}\right)_{K} \\
& =\sum_{K \in \Im_{h}} \delta_{K}\left(k \gamma j T_{h}^{n}+u_{h}^{n-1}, v_{h}-k \nu \triangle v_{h}+k\left(u_{h}^{n} \cdot \nabla\right) v_{h}+k \nabla q_{h}\right)_{K} \\
& +k \gamma\left(j T_{h}^{n}, v_{h}\right)+\left(u_{h}^{n-1}, v_{h}\right) \quad \forall\left(v_{h}, q_{h}\right) \in X_{h} \times M_{h}, \\
& \left(T_{h}^{n}, \phi_{h}\right)+k D\left(T_{h}^{n}, \phi_{h}\right)+k a_{2}\left(u_{h}^{n}, T_{h}^{n}, \phi_{h}\right)=\left(T_{h}^{n-1}, \phi_{h}\right) \quad \forall \phi_{h} \in W_{h}, \\
& u_{h}^{0}=0, \quad T_{h}^{0}=\psi(x, y) \quad \text { in } \Omega,
\end{aligned}
$$

where $\delta_{K}=\alpha h_{K}, \alpha>0$ is arbitrary constant.

Write $\hat{v}=(v, p)$ and $\hat{w}=(w, q)$. Define

$$
\begin{aligned}
& B_{\delta}\left(u, u_{h}^{n} ; \hat{v}, \hat{w}\right)=(v, w)+k a(v, w)+k a_{1}(u, v, w)-k b(p, w)+k b(q, v) \\
& +\sum_{K \in \Im_{h}} \delta_{K}\left(v-k \nu \triangle v+k(u \cdot \nabla) v+k \nabla p, w-k \nu \triangle w+k\left(u_{h}^{n} \cdot \nabla\right) w+k \nabla q\right)_{K}, \\
& F_{T \delta}(\hat{w})=k \gamma\left(j T_{h}^{n}, w\right)+\left(u_{h}^{n-1}, w\right) \\
& +\sum_{K \in \Im_{h}} \delta_{K}\left(u_{h}^{n-1}+k \gamma j T_{h}^{n}, w-k \nu \triangle w+k\left(u_{h}^{n} \cdot \nabla\right) w+k \nabla q\right)_{K}, \\
& \tilde{D}(v ; T, \phi)=(T, \phi)+k D(T, \phi)+k a_{2}(v, T, \phi) .
\end{aligned}
$$

Then Problem (III) could be rewritten as follows.

Problem (IV) Find $\hat{u}_{h}^{n} \equiv\left(u_{h}^{n}, p_{h}^{n}\right) \in X_{h} \times M_{h}$ such that, for $1 \leq n \leq N$,

$$
\begin{aligned}
& B_{\delta}\left(u_{h}^{n}, u_{h}^{n} ; \hat{u}_{h}^{n}, \hat{w}_{h}\right)=F_{T \delta}\left(\hat{w}_{h}\right) \quad \forall \hat{w}_{h} \equiv\left(v_{h}, q_{h}\right) \in X_{h} \times M_{h}, \\
& \tilde{D}\left(u_{h}^{n} ; T_{h}^{n}, \phi_{h}\right)=\left(T_{h}^{n-1}, \phi_{h}\right) \forall \phi_{h} \in W_{h}, \\
& u_{h}^{0}=0, \quad T_{h}^{0}=\psi(x, y) \quad \text { in } \Omega,
\end{aligned}
$$

where $\left.\delta\right|_{K}=\delta_{k}$. 
Throughout this paper, $C$ indicates a positive constant, and it is possibly different at different occurrences, which is independent of the mesh parameters $h$ and time step increment $k$, but may depend on $\Omega$, the Reynolds number, and on other parameters introduced in this paper.

The following properties for trilinear forms $a_{1}(\cdot, \cdot, \cdot)$ and $a_{2}(\cdot, \cdot, \cdot)$ are often used (see [35]).

$$
\begin{aligned}
& a_{1}(u, v, w)=-a_{1}(u, w, v), \quad a_{1}(u, v, v)=0 \quad \forall u, v, w \in X, \\
& \left|a_{1}(u, v, w)\right| \leq C_{1}\|u\|_{0}^{\frac{1}{2}}|u|_{1}^{\frac{1}{2}}\left(|v|_{1}^{\frac{1}{2}}\|v\|_{0}^{\frac{1}{2}}|w|_{1}+|v|_{1}\|w\|_{0}^{\frac{1}{2}}|w|_{1}^{\frac{1}{2}}\right) \quad \forall u, v, w \in X, \\
& a_{2}(u, T, \phi)=-a_{2}(u, \phi, T), \quad a_{2}(u, \phi, \phi)=0 \quad \forall u \in X, \forall T, \phi \in W, \\
& \left|a_{2}(u, T, \phi)\right| \leq C_{2}\|u\|_{0}^{\frac{1}{2}}|u|_{1}^{\frac{1}{2}}\left(|T|_{1}^{\frac{1}{2}}\|T\|_{0}^{\frac{1}{2}}|\phi|_{1}+|T|_{1}\|\phi\|_{0}^{\frac{1}{2}}|\phi|_{1}^{\frac{1}{2}}\right) \quad \forall u \in X, \forall T, \phi \in W,
\end{aligned}
$$

where $C_{1}$ is a constant independent of $u, v$, and $w$, and $C_{2}$ is a constant independent of $u, T$, and $\phi$. The bilinear forms $a(\cdot, \cdot), D(\cdot, \cdot)$, and $b(\cdot, \cdot)$ have the following properties

$$
\begin{gathered}
a(v, v) \geq \nu|v|_{1}^{2} \quad \forall v \in X,|a(u, v)| \leq \nu|u|_{1}|v|_{1} \quad \forall u, v \in X, \\
D(\phi, \phi) \geq \gamma^{-1}|\phi|_{1}^{2} \quad \forall \phi \in W,|D(T, \phi)| \leq \gamma^{-1}|T|_{1}|\phi|_{1} \quad \forall T, \phi \in W, \\
\sup _{v \in X} \frac{b(q, v)}{|v|_{1}} \geq \beta\|q\|_{0} \quad \forall q \in M,
\end{gathered}
$$

where $\beta$ is a constant. Define

$$
N_{0}=\sup _{u, v, w \in X} \frac{a_{1}(u, v, w)}{|u|_{1} \cdot|v|_{1} \cdot|w|_{1}}, \quad \tilde{N}_{0}=\sup _{u \in X,(T, \phi) \in W \times W} \frac{a_{2}(u, T, \phi)}{|u|_{1} \cdot|T|_{1} \cdot|\phi|_{1}} .
$$

The following discrete Gronwall lemma is well-known and very useful in context of next analysis (see $[4,34])$.

LEMma 2.2. If $\left\{a_{n}\right\},\left\{b_{n}\right\}$, and $\left\{c_{n}\right\}$ are three positive sequences, and $\left\{c_{n}\right\}$ is monotone, they satisfy

$$
a_{n}+b_{n} \leq c_{n}+\bar{\lambda} \sum_{i=0}^{n-1} a_{i}, \quad \bar{\lambda}>0, a_{0}+b_{0} \leq c_{0}
$$

then

$$
a_{n}+b_{n} \leq c_{n} \exp (n \bar{\lambda}), \quad n \geq 0 .
$$

For Problem (III) or (IV), we have the following result ${ }^{[13]}$.

THEOREM 2.3. If $h$ and $k$ are sufficiently small and $h=O(k)$, then there exists $h_{0}>0$ such that when $h<h_{0}$ Problem (III) has a unique solution sequence $\left(u_{h}^{n}, p_{h}^{n}, T_{h}^{n}\right) \in X_{h} \times M_{h} \times W_{h}$ and for $0 \leq n \leq N$,

$$
\begin{gathered}
\left\|u_{h}^{n}\right\|_{0}^{2}+k \sum_{i=1}^{n}\left\|u_{h}^{i}\right\|_{1}^{2}+\sum_{i=1}^{n}\left\|\delta^{\frac{1}{2}}\left(u_{h}^{i}-k \nu \Delta u_{h}^{i}+k\left(v_{h}^{i} \cdot \nabla\right) u_{h}^{i}+k \nabla p_{h}^{i}\right)\right\|_{0, h}^{2} \leq R M \\
\left\|u^{n}-u_{h}^{n}\right\|_{0}+(k \nu)^{\frac{1}{2}} \sum_{i=1}^{n}\left|u^{i}-u_{h}^{i}\right|_{1}+k^{\frac{1}{2}} \sum_{i=1}^{n}\left\|p^{i}-p_{h}^{i}\right\|_{0}+\left\|T^{n}-T_{h}^{n}\right\|_{0} \\
+\left(k \gamma^{-1}\right)^{\frac{1}{2}} \sum_{i=1}^{n}\left|T^{i}-T_{h}^{i}\right|_{1} \leq C\left(h^{\ell}+h^{\kappa}+h^{\iota}\right),
\end{gathered}
$$


where $M=t_{N}(R+2 k h \alpha) \gamma^{2}\|\varphi(x, y)\|_{0}^{2} \exp \left(2 \alpha h t_{N}\right), R=\nu^{-1},\|\cdot\|_{0, h}^{2}=\sum_{K \in \Im_{h}}\|\cdot\|_{0, K}^{2},\left(\hat{u}^{n}, T^{n}\right)=$ $\left(u^{n}, p^{n}, T^{n}\right) \in\left[W_{0}^{1, \infty}(\Omega) \cap H^{\ell+1}(\Omega)\right]^{2} \times H^{\kappa+1}(\Omega) \times\left[W_{0}^{1, \infty}(\Omega) \cap H^{\ell+1}(\Omega)\right]$ are the solutions for the problem (II), $C$ is the constant dependent on $\left|u^{n}\right|_{\ell+1},\left|p^{n}\right|_{\kappa}$, and $\left|T^{n}\right|_{\iota+1}$.

Combining Theorem 2.1 and Theorem 2.3 could yield the following result.

TheOREM 2.4. Under the assumptions of Theorem 2.1 and Theorem 2.3, there are the following error estimates, for $1 \leq n \leq N$,

$$
\begin{gathered}
\left\|u\left(t_{n}\right)-u_{h}^{n}\right\|_{0}+(k \nu)^{\frac{1}{2}} \sum_{i=1}^{n}\left|u\left(t_{i}\right)-u_{h}^{i}\right|_{1}+k^{\frac{1}{2}} \sum_{i=1}^{n}\left\|p\left(t_{i}\right)-p_{h}^{i}\right\|_{0} \\
+\left\|T\left(t_{n}\right)-T_{h}^{n}\right\|_{0}+(k \gamma-1)^{\frac{1}{2}} \sum_{i=1}^{n}\left|T\left(t_{i}\right)-T_{h}^{i}\right|_{1} \leq C\left(k+h^{\ell}+h^{\kappa}+h^{\iota}\right) .
\end{gathered}
$$

If $R=\nu^{-1}, \gamma$, triangulation parameter $h$, finite elements $X_{h}, M_{h}$, and $W_{h}$ and the time step increment $k$ are given, by solving Problem (III), we can obtain solution ensemble $\left\{u_{1 h}^{n}, u_{2 h}^{n}, p_{h}^{n}, T_{h}^{n}\right\}_{n=1}^{N}$ for Problem (III). And then we choose $L$ (for example, $L=20, N=200$, in general, $L \ll N)$ instantaneous solutions $U_{i}(x, y)=\left(u_{1 h}^{i}, u_{2 h}^{i}, p_{h}^{i}, T_{h}^{i}\right)(i=1,2, \cdots, L)$ (which are useful and of interest for us) from the $N$ groups of solutions $\left(u_{1 h}^{n}, u_{2 h}^{n}, p_{h}^{n}, T_{h}^{n}\right)(1 \leq n \leq N)$ for Problem (III), which are known as snapshots.

\section{Optimizing reduced PLSMFE formulation based POD technique for the non-stationary Navier-Stokes equations}

In this section, we use POD technique to deal with the snapshots in section 2 and develop an optimizing reduced PLSMFE formulation for the non-stationary Navier-Stokes equations.

Let $\hat{X}=X \times M \times W$. For $U_{i}(x, y)=\left(u_{1 h}^{i}, u_{2 h}^{i}, p_{h}^{i}, T_{h}^{i}\right)(i=1,2, \cdots, L)$ in section 2 , we set

$$
\mathcal{V}=\operatorname{span}\left\{U_{1}, U_{2}, \cdots, U_{L}\right\}
$$

and refer to $\mathcal{V}$ as the ensemble consisting of the snapshots $\left\{U_{i}\right\}_{i=1}^{L}$ at least one of which is supposed to be non-zero. Let $\left\{\psi_{j}\right\}_{j=1}^{l}$ denote an orthogonal basis of $\mathcal{V}$ with $l=\operatorname{dim} \mathcal{V}$. Then each member of the ensemble can be expressed as

$$
U_{i}=\sum_{j=1}^{l}\left(U_{i}, \psi_{j}\right)_{\hat{X}} \psi_{j} \text { for } i=1,2, \cdots, L,
$$

where $\left(U_{i}, \psi_{j}\right)_{\hat{X}}=\left(u_{h}^{i}, \psi_{u j}\right)_{X}+\left(p_{h}^{i}, \psi_{p j}\right)_{0},(\cdot, \cdot)_{0}$ is $L^{2}$-inner production, $\psi_{u j}$ and $\psi_{p j}$ are orthogonal bases corresponding to $u$ and $p$, respectively.

The method of POD consists in finding the orthogonal basis such that for every $d(1 \leq d \leq l)$ the mean square error between the elements $U_{i}(1 \leq i \leq L)$ and corresponding $d$-th partial sum of (3.2) is minimized on average:

$$
\min _{\left\{\psi_{j}\right\}_{j=1}^{d}} \frac{1}{L} \sum_{i=1}^{L}\left\|U_{i}-\sum_{j=1}^{d}\left(U_{i}, \psi_{j}\right)_{\hat{X}} \psi_{j}\right\|_{\hat{X}}
$$

such that

$$
\left(\psi_{i}, \psi_{j}\right)_{\hat{X}}=\delta_{i j} \text { for } 1 \leq i \leq d, 1 \leq j \leq i
$$


where $\left\|U_{i}\right\|_{\hat{X}}=\left[\left\|\nabla u_{1 h}^{i}\right\|_{0}^{2}+\left\|\nabla u_{2 h}^{i}\right\|_{0}^{2}+\left\|p_{h}^{i}\right\|_{0}^{2}+\left\|\nabla T_{h}^{i}\right\|_{0}^{2}\right]^{\frac{1}{2}}$. A solution $\left\{\psi_{j}\right\}_{j=1}^{d}$ of (3.3) and (3.4) is known as a POD basis of rank $d$.

We introduce the correlation matrix $K=\left(K_{i j}\right)_{L \times L} \in R^{L \times L}$ corresponding to the snapshots $\left\{U_{i}\right\}_{i=1}^{L}$ by

$$
K_{i j}=\frac{1}{L}\left(U_{i}, U_{j}\right)_{\hat{X}}
$$

The matrix $K$ is positive semi-definite and has rank $l$. The solution of (3.3) and (3.4) can be found in $[16,19,31]$, for example.

Proposition 3.1. Let $\lambda_{1} \geq \lambda_{2} \geq \cdots \geq \lambda_{l}>0$ denote the positive eigenvalues of $K$ and $v_{1}$, $v_{2}, \cdots, v_{l}$ the associated eigenvectors. Then a POD basis of rank $d \leq l$ is given by

$$
\psi_{i}=\frac{1}{\sqrt{\lambda_{i}}} v_{i}^{T}\left(U_{1}, U_{2}, \cdots, U_{L}\right)^{T}=\frac{1}{\sqrt{\lambda_{i}}} \sum_{j=1}^{L}\left(v_{i}\right)_{j} U_{j},
$$

where $\left(v_{i}\right)_{j}$ denotes the $j$-th component of the eigenvector $v_{i}$. Furthermore, the following error formula holds

$$
\frac{1}{L} \sum_{i=1}^{L}\left\|U_{i}-\sum_{j=1}^{d}\left(U_{i}, \psi_{j}\right)_{\hat{X}} \psi_{j}\right\|_{\hat{X}}=\sum_{j=d+1}^{l} \lambda_{j} .
$$

Let $\mathcal{V}^{d}=\operatorname{span}\left\{\psi_{1}, \psi_{2}, \cdots, \psi_{d}\right\}$ and $X^{d} \times M^{d} \times W^{d}=\mathcal{V}^{d}$ with $X^{d} \subset X, M^{d} \subset M$, and $W^{d} \subset W$. Set the Ritz-projection $P^{d}: X \rightarrow X^{d}, L^{2}$-projection $\rho^{d}: M \rightarrow M^{d}$, and Ritzprojection $\varrho^{d}: W \rightarrow W^{d}$ denoted by, respectively,

$$
\begin{array}{lc}
a\left(P^{d} u, v_{d}\right)=a\left(u, v_{d}\right) & \forall v_{d} \in X^{d}, \\
\left(\rho^{d} p, q_{d}\right)_{0}=\left(p, q_{d}\right)_{0} & \forall q_{d} \in M^{d}, \\
D\left(\varrho^{d} w, w^{d}\right)=D\left(w, w^{d}\right) & \forall w_{d} \in W^{d},
\end{array}
$$

where $u \in X, p \in M$, and $w \in W$. Due to (3.8) the linear operators $P^{d}$ and $\rho^{d}$ are well-defined and bounded:

$$
\begin{array}{ll}
\left\|\nabla\left(P^{d} u\right)\right\|_{0} \leq\|\nabla u\|_{0} & \forall u \in X, \\
\left\|\rho^{d} p\right\|_{0} \leq\|p\|_{0} & \forall p \in M, \\
\left\|\nabla\left(\varrho^{d} w\right)\right\|_{0} \leq\|\nabla w\|_{0} & \forall w \in W .
\end{array}
$$

Lemma 3.2. For every $d(1 \leq d \leq l)$ the projection operators $P^{d}, \rho^{d}$, and $\varrho^{d}$ satisfy respectively

$$
\begin{aligned}
& \frac{1}{L} \sum_{i=1}^{L}\left\|\nabla\left(u_{h}^{i}-P^{d} u_{h}^{i}\right)\right\|_{0} \leq \sum_{j=d+1}^{l} \lambda_{j}, \\
& \frac{1}{L} \sum_{i=1}^{L}\left\|p_{h}^{i}-\rho^{d} p_{h}^{i}\right\|_{0} \leq \sum_{j=d+1}^{l} \lambda_{j}, \\
& \frac{1}{L} \sum_{i=1}^{L}\left\|\nabla\left(T_{h}^{i}-P^{d} T_{h}^{i}\right)\right\|_{0} \leq \sum_{j=d+1}^{l} \lambda_{j},
\end{aligned}
$$


An optimizing reduced PLSMFE formulation

Proof. For any $u \in X$ we deduce from (3.8) that

$$
\begin{aligned}
\nu\left\|\nabla\left(u_{h}^{i}-P^{d} u_{h}^{i}\right)\right\|_{0}^{2} & =a\left(u_{h}^{i}-P^{d} u_{h}^{i}, u_{h}^{i}-P^{d} u_{h}^{i}\right)=a\left(u_{h}^{i}-P^{d} u_{h}^{i}, u_{h}^{i}-v_{d}\right) \\
& \leq \nu\left\|\nabla\left(u_{h}^{i}-P^{d} u_{h}^{i}\right)\right\|_{0}\left\|\nabla\left(u_{h}^{i}-v_{d}\right)\right\|_{0} \quad \forall v_{d} \in X^{d} .
\end{aligned}
$$

Furthermore,

$$
\left\|\nabla\left(u_{h}^{i}-P^{d} u_{h}^{i}\right)\right\|_{0} \leq\left\|\nabla\left(u_{h}^{i}-v_{d}\right)\right\|_{0} \quad \forall v_{d} \in X^{d} .
$$

Taking $v_{d}=\sum_{j=1}^{d}\left(u_{h}^{i}, \psi_{u j}\right)_{X} \psi_{u j}$ (where $\psi_{u j}$ is the component of $\psi_{j}$ corresponding to $u$ ) in (3.11), we can obtain the first inequality of (3.10) from (3.7).

Using Hölder inequality and the second inequality of (3.8) can yield

$$
\begin{aligned}
\left\|p_{h}^{i}-\rho^{d} p_{h}^{i}\right\|_{0}^{2} & =\left(p_{h}^{i}-\rho^{d} p_{h}^{i}, p_{h}^{i}-\rho^{d} p_{h}^{i}\right)=\left(p_{h}^{i}-\rho^{d} p_{h}^{i}, p_{h}^{i}-q_{d}\right) \\
& \leq\left\|p_{h}^{i}-\rho^{d} p_{h}^{i}\right\|_{0}\left\|p_{h}^{i}-q_{d}\right\|_{0} \quad \forall q_{d} \in M^{d},
\end{aligned}
$$

consequently,

$$
\left\|p_{h}^{i}-\rho^{d} p_{h}^{i}\right\|_{0} \leq\left\|p_{h}^{i}-q_{d}\right\|_{0} \quad \forall q_{d} \in M^{d}
$$

Taking $q_{d}=\sum_{j=1}^{d}\left(p_{h}^{i}, \psi_{p j}\right)_{0} \psi_{p j}$ (where $\psi_{p j}$ is the component of $\psi_{j}$ corresponding to $p$ ) in (3.12), from (3.7) we can obtain the second inequality of (3.10). Using the same technique as the first inequality of (3.10) can prove the third inequality of (3.10), which completes the proof of Lemma 3.2.

Thus, using $\mathcal{V}^{d}=X^{d} \times M^{d} \times W^{d}$, we can obtain the optimizing reduced PLSMFE formulation for Problem (IV) as follows.

Problem (V) Find $\left(\hat{u}_{d}^{n}, T_{d}^{n}\right) \equiv\left(u_{d}^{n}, p_{d}^{n}, T_{d}^{n}\right) \in \mathcal{V}^{d}$ such that

$$
\left\{\begin{array}{l}
B_{\delta}\left(u_{d}^{n}, u_{d}^{n} ; \hat{u}_{d}^{n}, \hat{w}_{d}^{n}\right)=F_{T \delta}\left(\hat{w}_{d}^{n}\right) \quad \forall \hat{w}_{d}^{n} \equiv\left(v_{d}, q_{d}\right) \in X^{d} \times M^{d}, \\
\tilde{D}\left(u_{d}^{n} ; T_{d}^{n}, \phi_{d}\right)=\left(T_{d}^{n-1}, \phi_{d}\right) \quad \forall \phi_{d} \in W^{d}, \\
u_{d}^{0}=0, T_{d}^{0}=\psi(x, y) \quad(x, y) \in \Omega,
\end{array}\right.
$$

where $1 \leq n \leq L$.

Remark 1. Problem (V) is an optimizing reduced PLSMFE formulation based on POD technique for Problem (IV), since it only includes $4 d$ degrees of freedom while Problem (IV) includes $4 N_{p}$ if $\kappa=\ell=\iota=1$ (where $N_{p}$ is the number of the vertices in $\Im_{h}$ ), Problem (IV) includes $4 N_{p}+4 N_{s} \approx 16 N_{p}$ if $\kappa=\ell=\iota=2$ and $4 d \ll 4 N_{p} \ll 16 N_{p}$ (where $N_{s}$ is the number of the sides in $\Im_{h}$ ). And since the residual is introduced, the combination of finite element subsets need not satisfy the BB stability condition and optimizing order error estimates will be obtained (see section 4). When one computes real-life problems, one may obtain the ensemble of snapshots from physical system trajectories by drawing samples from experiments and interpolation (or data assimilation). For example, for weather forecast, one can use previous weather prediction results to construct the ensemble of snapshots, then restructure the POD 
basis for the ensemble of snapshots by above (3.3)-(3.6), and finally combine it with a PetrovGalerkin least squares projection to derive an optimizing reduced order dynamical system, i.e., one needs only to solve the above Problem (V) which has only few degrees of freedom, but it is unnecessary to solve Problem (IV). Thus, the forecast of future weather change can be quickly simulated, which is of major importance for actual real-life applications.

\section{Existence and error analysis of solution of the optimizing reduced PLSMFE formulation for the non-stationary conduction-convection problems}

This section is devoted to discussing the existence and error estimates of solutions for Problem (V).

We see from (3.6) that $\mathcal{V}^{d}=X^{d} \times M^{d} \times W^{d} \subset \mathcal{V} \subset X_{h} \times M_{h} \times W_{h} \subset X \times M \times W$.

We first derive the following existence result for solutions of Problem (V).

Theorem 4.1. Under the assumptions of Theorem 2.1 and Theorem 2.3, Problem (V) has a unique solution sequence $\left(u_{d}^{n}, p_{d}^{n}\right) \in X^{d} \times M^{d}$ and satisfies, for $1 \leq n \leq N$,

$$
\left[\left\|u_{d}^{n}\right\|_{0}^{2}+k \sum_{i=1}^{n}\left\|u_{d}^{i}\right\|_{1}^{2}+\left\|\delta^{1 / 2}\left(u_{d}^{n}-k \nu \Delta u_{d}^{n}+k u_{d}^{n} \nabla u_{d}^{n}+k \nabla p_{d}^{n}\right)\right\|_{0, h}^{2}\right]^{1 / 2} \leq \sqrt{R M}
$$

Proof. We use Brouwer's fixed point theorem to prove our theorem.

For all $v_{d}^{n} \in X_{d}$ and $\left\|v_{d}^{n}\right\|_{0}^{2}+k \sum_{i=1}^{n}\left\|v_{d}^{i}\right\|_{1}^{2} \leq R M$, consider the following linearized Problem:

$$
\begin{aligned}
& B_{\delta}\left(v_{d}^{n}, v_{d}^{n} ; \hat{u}_{d}^{n}, \hat{w}_{d}\right)=F_{\delta}\left(\hat{w}_{d}\right) \quad \forall \hat{w}_{d} \in X^{d} \times M^{d}, \\
& u_{d}^{0}=0, \quad \text { in } \Omega, \\
& \tilde{D}\left(v_{d}^{n} ; T_{d}^{n}, \phi_{d}\right)=\left(T_{d}^{n-1}, \phi_{d}\right) \quad \forall \phi_{d} \in W^{d}, \\
& T_{d}^{0}=\psi(x, y) \quad \text { in } \Omega,
\end{aligned}
$$

Since $\tilde{D}\left(v_{d}^{n} ; \cdot, \cdot\right)$ is a coercive bilinear functional, linearized problem (4.3) has unique a group of solutions $T_{d}^{n} \in W^{d}(n=1,2, \cdots, N)$. For known $T_{d}^{n}$, since $B_{\delta}\left(v_{d}^{n}, v_{d}^{n} ; \cdot, \cdot\right)$ is a coercive bilinear functional, linearized problem (4.2) has unique a group of solutions $\hat{u}_{d}^{n}=\left(u_{d}^{n}, p_{d}^{n}\right)$ $(n=1,2, \cdots, N)$. Thus, there exists a map $G:\left(\hat{v}_{d}^{n}, \chi_{d}^{n}\right) \rightarrow\left(\hat{u}_{d}^{n}, T_{d}^{n}\right)(n=1,2, \cdots, N)$, where $\hat{v}_{d}^{n}=\left(v_{d}^{n}, \psi_{d}^{n}\right)$.

Taking $\phi_{d}=T_{d}^{n}$ in (4.3), from (2.6) we obtain that

$$
\left\|T_{d}^{n}\right\|_{0}^{2}+2 \gamma^{-1} k\left\|\nabla T_{d}^{n}\right\|_{0}^{2} \leq\left\|T_{d}^{n-1}\right\|_{0}^{2}
$$

Summing (4.4) from 1 to $n$ yields that

$$
\left\|T_{d}^{n}\right\|_{0}^{2}+\gamma^{-1} k \sum_{i=1}^{n}\left\|\nabla T_{d}^{i}\right\|_{0}^{2} \leq\|\psi(x, y)\|_{0}^{2} .
$$


An optimizing reduced PLSMFE formulation

Taking $\hat{w}_{d}=\hat{u}_{d}^{n}$ in (4.2), we obtain that

$$
\begin{aligned}
& \left\|u_{d}^{n}\right\|_{0}^{2}+k \nu\left|u_{d}^{n}\right|_{1}^{2}+\left\|\delta^{\frac{1}{2}}\left(u_{d}^{n}-k \nu \Delta u_{d}^{n}+k\left(v_{d}^{n} \cdot \nabla\right) u_{d}^{n}+k \nabla p_{d}^{n}\right)\right\|_{0, h}^{2} \\
& =\sum_{K \in \Im_{h}} \delta_{K}\left(k \gamma j T_{d}^{n}+u_{d}^{n-1}, u_{d}^{n}-k \nu \Delta u_{d}^{n}+k\left(v_{d}^{n} \cdot \nabla\right) u_{d}^{n}+k \nabla p_{d}^{n}\right)_{K} \\
& \quad+k \gamma j\left(T_{d}^{n}, u_{d}^{n}\right)+\left(u_{d}^{n-1}, u_{d}^{n}\right) \\
& \leq \frac{1}{2}\left(k R \gamma^{2}\left\|T_{d}^{n}\right\|_{-1}^{2}+k \nu\left|u_{d}^{n}\right|_{1}^{2}\right)+\frac{1}{2}\left(\left\|u_{d}^{n}\right\|_{0}^{2}+\left\|u_{d}^{n-1}\right\|_{0}^{2}\right) \\
& \quad+\frac{1}{2}\left\|\delta^{\frac{1}{2}}\left(k \gamma T_{d}^{n}+u_{d}^{n-1}\right)\right\|_{0}^{2}+\frac{1}{2}\left\|\delta^{\frac{1}{2}}\left(u_{d}^{n}-k \nu \Delta u_{d}^{n}+k\left(v_{d}^{n} \cdot \nabla\right) u_{d}^{n}+k \nabla p_{d}^{n}\right)\right\|_{0, h}^{2} .
\end{aligned}
$$

Noting that $\|\cdot\|_{-1} \leq\|\cdot\|_{0}$. From (4.6) we have

$$
\begin{aligned}
& \left\|u_{d}^{n}\right\|_{0}^{2}+k \nu\left|u_{d}^{n}\right|_{1}^{2}+\left\|\delta^{\frac{1}{2}}\left(u_{d}^{n}-k \nu \Delta u_{d}^{n}+k\left(v_{d}^{n} \cdot \nabla\right) u_{d}^{n}+k \nabla p_{d}^{n}\right)\right\|_{0, h}^{2} \\
& \leq k R \gamma^{2}\left\|T_{d}^{n}\right\|_{0}^{2}+\left\|u_{d}^{n-1}\right\|_{0}^{2}+2\left\|\delta^{\frac{1}{2}} k \gamma T_{d}^{n}\right\|_{0}^{2}+2 \alpha h\left\|u_{d}^{n-1}\right\|_{0}^{2} \\
& \leq k(R+2 k h \alpha) \gamma^{2}\|\varphi(x, y)\|_{0}^{2}+\left\|u_{d}^{n-1}\right\|_{0}^{2}+2 \alpha h\left\|u_{d}^{n-1}\right\|_{0}^{2} .
\end{aligned}
$$

Summing (4.7) from 1 to $n$ and noting that $u_{d}^{0}=0$ could yield

$$
\begin{aligned}
& \left\|u_{d}^{n}\right\|_{0}^{2}+k \nu \sum_{i=1}^{n}\left|u_{d}^{i}\right|_{1}^{2}+\sum_{i=1}^{n}\left\|\delta^{\frac{1}{2}}\left(u_{d}^{i}-k \nu \Delta u_{d}^{i}+k\left(v_{d}^{i} \cdot \nabla\right) u_{d}^{i}+k \nabla p_{d}^{i}\right)\right\|_{0, h}^{2} \\
& \leq n k(R+2 k h \alpha) \gamma^{2}\|\varphi(x, y)\|_{0}^{2}+2 \alpha h \sum_{i=0}^{n-1}\left\|u_{d}^{i}\right\|_{0}^{2} .
\end{aligned}
$$

By discrete Gronwall inequality we get that

$$
\begin{aligned}
& \left\|u_{d}^{n}\right\|_{0}^{2}+k \nu \sum_{i=1}^{n}\left|u_{d}^{i}\right|_{1}^{2}+\sum_{i=1}^{n}\left\|\delta^{\frac{1}{2}}\left(u_{d}^{i}-k \nu \Delta u_{d}^{i}+k\left(v_{d}^{i} \cdot \nabla\right) u_{d}^{i}+k \nabla p_{d}^{i}\right)\right\|_{0, h}^{2} \\
& \leq n k(R+2 k h \alpha) \gamma^{2}\|\varphi(x, y)\|_{0}^{2} \exp (2 \alpha h n) .
\end{aligned}
$$

Note that $1<\nu^{-1}$ and $k n \leq t_{N}$. From (4.9) we obtain that

$$
\begin{aligned}
& \left\|u_{d}^{n}\right\|_{0}^{2}+k \sum_{i=1}^{n}\left|u_{d}^{i}\right|_{1}^{2}+\sum_{i=1}^{n}\left\|\delta^{\frac{1}{2}}\left(u_{d}^{i}-k \nu \Delta u_{d}^{i}+k\left(v_{d}^{i} \cdot \nabla\right) u_{d}^{i}+k \nabla p_{d}^{i}\right)\right\|_{0, h}^{2} \\
& \leq R t_{N}(R+2 k h \alpha) \gamma^{2}\|\varphi(x, y)\|_{0}^{2} \exp \left(2 \alpha t_{N}\right) \equiv R M .
\end{aligned}
$$

Let $B_{R M}=\left\{\left(v_{d}^{n}, \psi_{d}^{n}, \chi_{d}^{n}\right) \in X^{d} \times M^{d} \times W^{d} ;\left\|v_{d}^{n}\right\|_{0}^{2}+k \sum_{i=1}^{n}\left|v_{d}^{i}\right|_{1}^{2} \leq R M\right\}$. It is shown by (4.5) and (4.10) that the map $G: B_{R M} \rightarrow B_{R M}$. Thus, it is necessary to prove that $F$ is continuous. For any $\left(\hat{v}_{d}^{1 n}, \chi_{d}^{1 n}\right)=\left(v_{d}^{1 n}, \psi_{d}^{1 n}, \chi_{d}^{1 n}\right)$ and $\left(\hat{v}_{d}^{2 n}, \chi_{d}^{2 n}\right)=\left(v_{d}^{2 n}, \psi_{d}^{2 n}, \chi_{d}^{2 n}\right) \in B_{R M}$, by (4.2) and (4.3) we obtain two groups of solutions $\left(u_{d}^{1 n}, p_{d}^{1 n}, T_{d}^{1 n}\right)$ and $\left(u_{d}^{2 n}, p_{d}^{2 n}, T_{d}^{2 n}\right)(n=1,2, \cdots, N)$ such that

$$
\begin{aligned}
& B_{\delta}\left(v_{d}^{1 n}, v_{d}^{1 n} ; \hat{u}_{d}^{1 n}, \hat{w}_{d}\right)=F_{\delta}\left(\hat{w}_{d}\right) \quad \forall \hat{w}_{d} \in X^{d} \times M^{d}, \\
& \tilde{D}\left(v_{d}^{1 n} ; T_{d}^{1 n}, \phi_{d}\right)=\left(T_{d}^{1(n-1)}, \phi_{d}\right) \quad \forall \phi_{d} \in W^{d}, \\
& u_{d}^{10}=0, \quad T_{d}^{10}=\psi(x, y) \quad(x, y) \in \Omega, \\
& \left\|u^{1 n}\right\|_{0}^{2}+k \sum_{i=1}^{n}\left\|u_{d}^{1 i}\right\|_{1}^{2} \leq R M
\end{aligned}
$$


and

$$
\begin{aligned}
& B_{\delta}\left(v_{d}^{2 n}, v_{d}^{2 n} ; \hat{u}_{d}^{2 n}, \hat{w}_{d}\right)=F_{\delta}\left(\hat{w}_{d}\right) \quad \forall \hat{w}_{d} \in X^{d} \times M^{d}, \\
& D\left(v_{d}^{2 n} ; T_{d}^{2 n}, \phi_{d}\right)=\left(T_{d}^{2(n-1)}, \phi_{d}\right) \quad \forall \phi_{d} \in W^{d}, \\
& u_{d}^{20}=0, \quad T_{d}^{20}=\psi(x, y) \quad(x, y) \in \Omega, \\
& \left\|u^{2 n}\right\|_{0}^{2}+k \sum_{i=1}^{n}\left\|u_{d}^{2 i}\right\|_{1}^{2} \leq R M .
\end{aligned}
$$

By (4.11), (4.12), (2.6), (2.10), (4.5), and inverse inequality, we obtain that

$$
\begin{aligned}
& \left\|T_{d}^{1 n}-T_{d}^{2 n}\right\|_{0}^{2}+k \gamma^{-1}\left|T_{d}^{1 n}-T_{d}^{2 n}\right|_{1}^{2} \\
& =\left(T_{d}^{1(n-1)}-T_{d}^{2(n-1)}, T_{d}^{1 n}-T_{d}^{2 n}\right)-k a_{2}\left(v_{d}^{1 n}-v_{d}^{2 n}, T_{d}^{1 n}, T_{d}^{1 n}-T_{d}^{2 n}\right) \\
& \leq\left\|T_{d}^{1 n}-T_{d}^{2 n}\right\|_{0}\left\|T_{d}^{1(n-1)}-T_{d}^{2(n-1)}\right\|_{0}+C k\left|v_{d}^{1 n}-v_{d}^{2 n}\right|_{1}\left|T_{d}^{1 n}-T_{d}^{2 n}\right|_{1}\left\|T_{d}^{1 n}\right\|_{0} \\
& \leq \frac{1}{2}\left\|T_{d}^{1 n}-T_{d}^{2 n}\right\|_{0}^{2}+\frac{1}{2}\left\|T_{d}^{1(n-1)}-T_{d}^{2(n-1)}\right\|_{0}^{2} \\
& +C k\left|v_{d}^{1 n}-v_{d}^{2 n}\right|_{1}^{2}+\frac{1}{2} k \gamma^{-1}\left|T_{d}^{1 n}-T_{d}^{2 n}\right|_{1}^{2} .
\end{aligned}
$$

Therefore, we get that

$$
\left\|T_{d}^{1 n}-T_{d}^{2 n}\right\|_{0}^{2}+k \gamma^{-1}\left|T_{d}^{1 n}-T_{d}^{2 n}\right|_{1}^{2} \leq\left\|T_{d}^{1(n-1)}-T_{d}^{2(n-1)}\right\|_{0}^{2}+C k\left|v_{d}^{1 n}-v_{d}^{2 n}\right|_{1}^{2} .
$$

Summing (4.14) from 1 to $n$ can yield that

$$
\left\|T_{d}^{1 n}-T_{d}^{2 n}\right\|_{0}^{2}+k \gamma^{-1} \sum_{i=1}^{n}\left|T_{d}^{1 i}-T_{d}^{2 i}\right|_{1}^{2} \leq C k \sum_{i=1}^{n}\left|v_{d}^{1 i}-v_{d}^{2 i}\right|_{1}^{2} .
$$

By (4.11) and (4.12), we obtain that, $\forall \hat{w}_{d}=\left(w_{d}, r_{d}\right) \in X^{d} \times M^{d}$,

$$
\begin{aligned}
& B_{\delta}\left(v_{d}^{1 n}, v_{d}^{1 n}, \hat{u}_{d}^{1 n}, \hat{w}_{d}\right)-B_{\delta}\left(v_{d}^{2 n}, v_{d}^{2 n}, \hat{u}_{d}^{2 n}, \hat{w}_{d}\right) \\
& =\left(u_{d}^{1(n-1)}-u_{d}^{2(n-1)}, w_{d}\right)+\sum_{K \in \Im_{h}} \delta_{K}\left(u_{d}^{2(n-1)}, k\left(v_{d}^{1 n}-v_{d}^{2 n}\right) \cdot \nabla w_{d}\right)_{K} \\
& \quad+\sum_{K \in \Im_{h}} \delta_{K}\left(u_{d}^{1(n-1)}-u_{d}^{2(n-1)}, w_{d}-k \nu \Delta w_{d}+k v_{d}^{1 n} \cdot \nabla w_{d}+k \nabla r_{d}\right)_{K} \\
& \quad+\sum_{K \in \Im_{h}} \delta_{K}\left(k \gamma j\left(T_{d}^{1 n}-T_{d}^{2 n}\right), w_{d}-k \nu \Delta w_{d}+k v_{d}^{1 n} \cdot \nabla w_{d}+k \nabla r_{d}\right)_{K} \\
& \quad+k \gamma\left(j\left(T_{d}^{1 n}-T_{d}^{2 n}\right), w_{d}\right)+\sum_{K \in \Im_{d}} \delta_{K}\left(k \gamma j T_{d}^{2 n}, k\left(v_{d}^{1 n}-v_{d}^{2 n}\right) \nabla w_{d}\right) \equiv S_{0} .
\end{aligned}
$$

Taking $w_{d}=u_{d}^{1 n}-u_{d}^{2 n}$ and $r_{d}=p_{d}^{1 n}-p_{d}^{2 n}$, on the one hand, we get that

$$
\begin{aligned}
& B_{\delta}\left(v_{d}^{1 n}, v_{d}^{1 n}, \hat{w}_{d}, \hat{w}_{d}\right)=\left\|w_{d}\right\|_{0}^{2}+k \nu\left|w_{d}\right|_{1}^{2} \\
& +\left\|\delta^{\frac{1}{2}}\left(w_{d}-k \nu \Delta w_{d}+k v_{d}^{1 n} \cdot \nabla w_{d}+k \nabla r_{d}\right)\right\|_{0, h}^{2} .
\end{aligned}
$$


On the other hand, by (4.11) and (4.12) we obtain

$$
\begin{aligned}
& B_{\delta}\left(v_{d}^{1 n}, v_{d}^{1 n}, \hat{w}_{d}, \hat{w}_{d}\right)=B_{\delta}\left(v_{d}^{1 n}, v_{d}^{1 n}, \hat{u}_{d}^{1 n}, \hat{w}_{d}\right)-B_{\delta}\left(v_{d}^{1 n}, v_{d}^{1 n}, \hat{u}_{d}^{2 n}, \hat{w}_{d}\right) \\
= & B_{\delta}\left(v_{d}^{2 n}, v_{d}^{2 n}, \hat{u}_{d}^{2 n}, \hat{w}_{d}\right)-B_{\delta}\left(v_{d}^{1 n}, v_{d}^{1 n}, \hat{u}_{d}^{2 n}, \hat{w}_{d}\right)+S_{0}=k a_{1}\left(v_{d}^{2 n}-v_{d}^{1 n}, u_{d}^{2 n}, w_{d}\right) \\
& +\sum_{K \in \Im_{h}} \delta_{K}\left(k\left(v_{d}^{2 n}-v_{d}^{1 n}\right) \nabla u_{d}^{2 n}, w_{d}-k \nu \Delta w_{d}+k v_{d}^{1 n} \cdot \nabla w_{d}+k \nabla r_{d}\right)_{K} \\
& +\sum_{K \in \Im_{h}} \delta_{K}\left(u_{d}^{2 n}-k \nu \Delta u_{d}^{2 n}+k v_{d}^{2 n} \cdot \nabla u_{d}^{2 n}+k \nabla p_{d}^{2 n}, k\left(v_{d}^{2 n}-v_{d}^{1 n}\right) \nabla w_{d}\right)_{K}+S_{0} \\
\equiv & S_{1}+S_{2}+S_{3}+S_{0} .
\end{aligned}
$$

By (2.10) and (4.12), we obtain that

$$
\left|S_{1}\right|=\left|k a_{1}\left(v_{d}^{2 n}-v_{d}^{1 n}, u_{d}^{2 n}, w_{d}\right)\right| \leq k R M N_{0}\left|v_{d}^{2 n}-v_{d}^{1 n}\right|_{1}\left|w_{d}\right|_{1} .
$$

By inverse inequality $\left\|v_{d}\right\|_{0, \infty} \leq C\left|v_{d}\right|_{1}$ and $\left\|v_{d}\right\|_{0} \leq C h\left|v_{d}\right|_{1}$ (for all $v_{d} \in X^{d} \subset X_{h}$, see [35-36]), (2.10), (4.12), and (4.13), we obtain that

$$
\begin{aligned}
& \left|S_{2}\right|=\left|\sum_{K \in \Im_{h}} \delta_{K}\left(k\left(v_{d}^{2 n}-v_{d}^{1 n}\right) \nabla u_{d}^{2 n}, w_{d}-k \nu \Delta w_{d}+k v_{d}^{1 n} \cdot \nabla w_{d}+k \nabla r_{d}\right)_{K}\right| \\
& \leq C k h^{\frac{1}{2}}\left\|v_{d}^{2 n}-v_{d}^{1 n}\right\|_{1}\left\|\delta^{\frac{1}{2}}\left(w_{d}-k \nu \Delta w_{d}+k v_{d}^{1 n} \cdot \nabla w_{d}+k \nabla r_{d}\right)\right\|_{0, h}, \\
& \left|S_{3}\right|=\left|\sum_{K \in \Im_{h}} \delta_{K}\left(u_{d}^{2 n}-k \nu \Delta u_{d}^{2 n}+k v_{d}^{2 n} \cdot \nabla u_{d}^{2 n}+k \nabla p_{d}^{2 n}, k\left(v_{d}^{2 n}-v_{d}^{1 n}\right) \nabla w_{d}\right)_{K}\right| \\
& \leq C k h^{\frac{1}{2}}\left|v_{d}^{2 n}-v_{d}^{1 n}\right|_{1}\left|w_{d}\right|_{1} \\
& \left|S_{0}\right|=\mid\left(u_{d}^{1(n-1)}-u_{d}^{2(n-1)}, w_{d}\right)+\sum_{K \in \Im_{h}} \delta_{K}\left(u_{d}^{2(n-1)}, k\left(v_{d}^{1 n}-v_{d}^{2 n}\right) \cdot \nabla w_{d}\right)_{K} \\
& +\sum_{K \in \Im_{h}} \delta_{K}\left(u_{d}^{1(n-1)}-u_{d}^{2(n-1)}, w_{d}-k \nu \Delta w_{d}+k v_{d}^{1 n} \cdot \nabla w_{d}+k \nabla r_{d}\right)_{K} \\
& +\sum_{K \in \Im_{h}} \delta_{K}\left(k \gamma j\left(T_{d}^{1 n}-T_{d}^{2 n}\right), w_{d}-k \nu \Delta w_{d}+k v_{d}^{1 n} \cdot \nabla w_{d}+k \nabla r_{d}\right)_{K} \\
& +k \gamma\left(j\left(T_{d}^{1 n}-T_{d}^{2 n}\right), w_{d}\right)+\sum_{K \in \Im_{h}} \delta_{K}\left(\gamma j T_{d}^{2 n}, k\left(v_{d}^{1 n}-v_{d}^{2 n}\right) \nabla w_{d}\right) \mid \\
& \leq\left\|u_{d}^{1(n-1)}-u_{d}^{2(n-1)}\right\|_{0}\left\|w_{d}\right\|_{0}+C k h\left|v_{d}^{1 n}-v_{d}^{2 n}\right|_{1}\left|w_{d}\right|_{1} \\
& +C h^{\frac{1}{2}}\left\|u_{d}^{1(n-1)}-u_{d}^{2(n-1)}\right\|_{0}\left\|\delta^{\frac{1}{2}}\left(w_{d}-k \nu \Delta w_{d}+k v_{d}^{1 n} \cdot \nabla w_{d}+k \nabla r_{d}\right)\right\|_{0, h} \\
& +C k h^{\frac{1}{2}}\left\|T_{d}^{1 n}-T_{d}^{2 n}\right\|_{0}\left\|\delta^{\frac{1}{2}}\left(w_{d}-k \nu \Delta w_{d}+k v_{d}^{1 n} \cdot \nabla w_{d}+k \nabla r_{d}\right)\right\|_{0, h} \\
& +k h \gamma\left\|T_{d}^{1 n}-T_{d}^{2 n}\right\|_{0}\left\|w_{d}\right\|_{1}+C h k \gamma\left|v_{d}^{1 n}-v_{d}^{2 n}\right|_{1}\left|w_{d}\right|_{1} .
\end{aligned}
$$

If $h=O(k)$, combining (4.19)-(4.22) and (4.15) and using Cauchy inequality could yield that

$$
\begin{aligned}
& \left|S_{0}\right|+\left|S_{1}\right|+\left|S_{2}\right|+\left|S_{3}\right| \leq \frac{1}{2}\left\|u_{d}^{1(n-1)}-u_{d}^{2(n-1)}\right\|_{0}^{2}+\frac{1}{2}\left\|w_{d}\right\|_{0} \\
& +C k^{2}\left|v_{d}^{1 n}-v_{d}^{2 n}\right|_{1}^{2}+\frac{k \nu}{2}\left|w_{d}\right|_{1}^{2}+C k\left\|u_{d}^{1(n-1)}-u_{d}^{2(n-1)}\right\|_{0}^{2} \\
& +\frac{1}{2}\left\|\delta^{\frac{1}{2}}\left(w_{d}-k \nu \Delta w_{d}+k v_{d}^{1 n} \cdot \nabla w_{d}+k \nabla r_{d}\right)\right\|_{0, h}^{2}+C k^{3} \sum_{i=1}^{n}\left|v_{d}^{1 i}-v_{d}^{2 i}\right|_{1}^{2} .
\end{aligned}
$$


Combining (4.23) and (4.17)-(4.18) yields

$$
\begin{aligned}
& \| \delta^{\frac{1}{2}}\left(u_{d}^{1 n}-u_{d}^{2 n}\right)-k \nu \Delta\left(u_{d}^{1 n}-u_{d}^{2 n}\right)+k v_{d}^{1 n} \cdot \nabla\left(u_{d}^{1 n}-u_{d}^{2 n}\right) \\
& +k \nabla\left(p_{d}^{1 n}-p_{d}^{2 n}\right)\left\|_{0, h}^{2}+\right\| u_{d}^{2 n}-u_{d}^{1 n} \|_{0}^{2}+k \nu\left|u_{d}^{2 n}-u_{d}^{1 n}\right|_{1}^{2} \\
& \leq\left\|u_{d}^{1(n-1)}-u_{d}^{2(n-1)}\right\|_{0}^{2}+C k\left\|u_{d}^{1(n-1)}-u_{d}^{2(n-1)}\right\|_{0}^{2}+C k^{3} \sum_{i=1}^{n}\left|v_{d}^{1 i}-v_{d}^{2 i}\right|_{1}^{2} .
\end{aligned}
$$

Summing (4.24) from 1 to $n$ we get

$$
\begin{aligned}
& \sum_{i=1}^{n} \| \delta^{\frac{1}{2}}\left(u_{d}^{1 i}-u_{d}^{2 i}\right)-k \nu \Delta\left(u_{d}^{1 i}-u_{d}^{2 i}\right)+k v_{d}^{1 i} \cdot \nabla\left(u_{d}^{1 i}-u_{d}^{2 i}\right) \\
& +k \nabla\left(p_{d}^{1 i}-p_{d}^{2 i}\right)\left\|_{0, h}^{2}+\right\| u_{d}^{1 n}-u_{d}^{2 n} \|_{0}^{2}+k \nu \sum_{i=1}^{n}\left|u_{d}^{1 i}-u_{d}^{2 i}\right|_{1}^{2} \\
& \leq C k \sum_{i=0}^{n-1}\left\|u_{d}^{1 i}-u_{d}^{2 i}\right\|_{0}^{2}+C k^{3} n \sum_{i=1}^{n}\left|v_{d}^{1 i}-v_{d}^{2 i}\right|_{1}^{2} .
\end{aligned}
$$

By discrete Gronwall inequality, we obtain that

$$
\begin{aligned}
& \sum_{i=1}^{n}\left\|\delta^{\frac{1}{2}}\left(u_{d}^{1 i}-u_{d}^{2 i}\right)-k \nu \Delta\left(u_{d}^{1 i}-u_{d}^{2 i}\right)+k v_{d}^{1 i} \cdot \nabla\left(u_{d}^{1 i}-u_{d}^{2 i}\right)+k \nabla\left(p_{d}^{1 i}-p_{d}^{2 i}\right)\right\|_{0, h}^{2} \\
& +\left\|u_{d}^{1 n}-u_{d}^{2 n}\right\|_{0}^{2}+k \nu \sum_{i=1}^{n}\left|u_{d}^{1 i}-u_{d}^{2 i}\right|_{1}^{2} \leq C k^{3} n \sum_{i=1}^{n}\left|v_{d}^{1 i}-v_{d}^{2 i}\right|_{1}^{2} \exp (C n k) .
\end{aligned}
$$

Since $n k \leq t_{N}$, we obtain that

$$
\begin{aligned}
& \sum_{i=1}^{n}\left\|\delta^{\frac{1}{2}}\left(u_{d}^{1 i}-u_{d}^{2 i}\right)-k \nu \Delta\left(u_{d}^{1 i}-u_{d}^{2 i}\right)+k v_{d}^{1 i} \cdot \nabla\left(u_{d}^{1 i}-u_{d}^{2 i}\right)+k \nabla\left(p_{d}^{1 i}-p_{d}^{2 i}\right)\right\|_{0, h}^{2} \\
& +\left\|u_{d}^{1 n}-u_{d}^{2 n}\right\|_{0}^{2}+k \nu \sum_{i=1}^{n}\left|u_{d}^{1 i}-u_{d}^{2 i}\right|_{1}^{2} \leq C k^{2} \sum_{i=1}^{n}\left|v_{d}^{1 i}-v_{d}^{2 i}\right|_{1}^{2} .
\end{aligned}
$$

Thus, (4.15) and (4.27) show that the map $G: B_{R M} \rightarrow B_{R M}$ is continuous. By Brouwer's fixed point theorem, this implies that $G$ has at least one fixed $\left(\hat{u}_{d}^{n}, T_{d}^{n}\right)=G\left(\hat{u}_{d}^{n}, T_{d}^{n}\right)(n=1,2, \cdots, N)$, i.e., Problem (IV) has at least one solution sequence $\left(u_{d}^{n}, p_{d}^{n}, T_{d}^{n}\right) \in X^{d} \times M^{d} \times W^{d}$.

If $\left(u_{d}^{1 n}, p_{d}^{1 n}, T_{d}^{1 n}\right) \in X^{d} \times M^{d} \times W^{d}$ and $\left(u_{d}^{21 n}, p_{d}^{2 n}, T_{d}^{2 n}\right) \in X^{d} \times M^{d} \times W^{d}$ are two groups of solutions for Problem (IV), using the same approaches as in (4.15) and (4.27), we derive that

$$
\begin{gathered}
\left\|T_{d}^{1 n}-T_{d}^{2 n}\right\|_{0}^{2}+k \gamma^{-1} \sum_{i=1}^{n}\left|T_{d}^{1 i}-T_{d}^{2 i}\right|_{1}^{2} \leq C k \sum_{i=1}^{n}\left|u_{d}^{1 i}-u_{d}^{2 i}\right|_{1}^{2}, \\
\sum_{i=1}^{n}\left\|\delta^{\frac{1}{2}}\left(u_{d}^{1 i}-u_{d}^{2 i}\right)-k \nu \Delta\left(u_{d}^{1 i}-u_{d}^{2 i}\right)+k u_{d}^{1 i} \cdot \nabla\left(u_{d}^{1 i}-u_{d}^{2 i}\right)+k \nabla\left(p_{d}^{1 i}-p_{d}^{2 i}\right)\right\|_{0, h}^{2} \\
+\left\|u_{d}^{1 n}-u_{d}^{2 n}\right\|_{0}^{2}+k \nu \sum_{i=1}^{n}\left|u_{d}^{1 i}-u_{d}^{2 i}\right|_{1}^{2} \leq C k h \sum_{i=1}^{n}\left|u_{d}^{1 i}-u_{d}^{2 i}\right|_{1}^{2} .
\end{gathered}
$$

Therefore there is an $h_{0}=\nu /(2 C)$ such that if $h \leq h_{0}$, we get that

$$
\begin{aligned}
& \sum_{i=1}^{n}\left\|\delta^{\frac{1}{2}}\left(u_{d}^{1 i}-u_{d}^{2 i}\right)-k \nu \Delta\left(u_{d}^{1 i}-u_{d}^{2 i}\right)+k u_{d}^{1 i} \cdot \nabla\left(u_{d}^{1 i}-u_{d}^{2 i}\right)+k \nabla\left(p_{d}^{1 i}-p_{d}^{2 i}\right)\right\|_{0, h}^{2} \\
& +\left\|u_{d}^{1 n}-u_{d}^{2 n}\right\|_{0}^{2}+k \nu \sum_{i=1}^{n}\left|u_{d}^{1 i}-u_{d}^{2 i}\right|_{1}^{2} \leq 0,
\end{aligned}
$$


which shows that $u_{d}^{1 n}=u_{d}^{2 n}$ and $p_{d}^{1 n}=p_{d}^{2 n}$. And by (4.28) we get $T_{d}^{1 n}=T_{d}^{2 n}$. Therefore, the solutions $\left(u_{d}^{n}, p_{d}^{n}, T_{d}^{n}\right)(1 \leq n \leq N)$ for Problem (IV) are unique.

In the following theorem, the errors between the solutions $\left(u_{d}^{n}, p_{d}^{n}\right)$ for Problem (V) and the solutions $\left(u_{h}^{n}, p_{h}^{n}\right)$ for Problem (IV) are derived.

TheOrem 4.2. Under the assumptions of Theorem 2.1 and Theorem 2.3, if $h$ and $k$ are sufficiently small, $h=O(k)$, and $k=O\left(L^{-2}\right)$, then the errors between the solutions $\left(u_{d}^{n}, p_{d}^{n}, T_{d}^{n}\right)$ for Problem (V) and the solutions $\left(u_{h}^{n}, p_{h}^{n}, T_{h}^{n}\right)$ for Problem (IV) have the following error estimates, for $1 \leq n \leq N$,

$$
\begin{aligned}
& \left\|u_{h}^{n}-u_{d}^{n}\right\|_{0}+\left\|T_{h}^{n}-T_{d}^{n}\right\|_{0}+(k \nu)^{\frac{1}{2}} \sum_{i=1}^{n}\left\|\nabla\left(u_{h}^{i}-u_{d}^{i}\right)\right\|_{0}+k^{\frac{1}{2}} \sum_{i=1}^{n}\left\|p_{h}^{i}-p_{d}^{i}\right\|_{0} \\
& +\left(k \gamma^{-1}\right)^{\frac{1}{2}} \sum_{i=1}^{n}\left\|\nabla\left(T_{h}^{i}-T_{d}^{i}\right)\right\|_{0} \leq C \sum_{j=d+1}^{l} \lambda_{j}, \text { if } n \in\{1,2, \cdots, L\} ;
\end{aligned}
$$

and if snapshots are equably taken,

$$
\begin{aligned}
& \left\|u_{h}^{n}-u_{d}^{n}\right\|_{0}+\left\|T_{h}^{n}-T_{d}^{n}\right\|_{0}+(k \nu)^{\frac{1}{2}} \sum_{i=1}^{n}\left\|\nabla\left(u_{h}^{i}-u_{d}^{i}\right)\right\|_{0}+k^{\frac{1}{2}} \sum_{i=1}^{n}\left\|p_{h}^{i}-p_{d}^{i}\right\|_{0} \\
& +\left(k \gamma^{-1}\right)^{\frac{1}{2}} \sum_{i=1}^{n}\left\|\nabla\left(T_{h}^{i}-T_{d}^{i}\right)\right\|_{0} \leq C k \sqrt{\frac{N}{4 L}}+C \sum_{j=d+1}^{l} \lambda_{j}, \text { if } n \notin\{1,2, \cdots, L\} .
\end{aligned}
$$

Proof. Let $\hat{w}_{d}=\left(w_{d}^{n}, r_{d}^{n}\right), w_{d}^{n}=P^{d} u_{h}^{n}-u_{d}^{n}$, and $r_{d}^{n}=\rho^{d} p_{h}^{n}-p_{d}^{n}$. On the one hand, we have that

$$
\begin{aligned}
& B_{\delta}\left(u_{d}^{n}, u_{d}^{n}, \hat{w}_{d}, \hat{w}_{d}\right) \\
& =\left\|w_{d}^{n}\right\|_{0}^{2}+k \nu\left|w_{d}^{n}\right|_{1}^{2}+\left\|\delta^{\frac{1}{2}}\left(w_{d}^{n}-k \nu \Delta w_{d}^{n}+k u_{d}^{n} \nabla w_{d}^{n}+k \nabla r_{d}^{n}\right)\right\|_{0, h}^{2} .
\end{aligned}
$$

On the other hand, if write $\hat{P}^{d} \hat{u}=\left(P^{d} u_{h}^{n}, \rho^{d} p_{h}^{n}\right)$ and $\hat{u}_{d}=\left(u_{d}^{n}, p_{h}^{d}\right)$, we have that

$$
\begin{aligned}
& B_{\delta}\left(u_{d}^{n}, u_{d}^{n}, \hat{w}_{d}, \hat{w}_{d}\right)=B_{\delta}\left(u_{d}^{n}, u_{d}^{n}, \hat{P}^{d} \hat{u}, \hat{w}_{d}\right)-B_{\delta}\left(u_{d}^{n}, u_{d}^{n}, \hat{u}_{d}, \hat{w}_{d}\right) \\
& =B_{\delta}\left(u_{d}^{n}, u_{d}^{n}, \hat{P}^{d} \hat{u}, \hat{w}_{d}\right)-B_{\delta}\left(u_{h}^{n}, u_{d}^{n}, \hat{u}_{h}^{n}, \hat{w}_{d}\right)+\left(u_{h}^{n-1}-u_{d}^{n-1}, w_{d}^{n}\right) \\
& +\sum_{K \in \Im_{h}} \delta_{K}\left(u_{h}^{n-1}-u_{d}^{n-1}+k \gamma j\left(T_{h}^{n}-T_{d}^{n}\right), w_{d}^{n}-k \nu \Delta w_{d}^{n}+k u_{d}^{n} \nabla w_{d}^{n}+k \nabla r_{d}^{n}\right)_{K} \\
& +k \gamma j\left(T_{h}^{n}-T_{d}^{n}, w_{d}^{n}\right) \equiv \bar{S}_{1}+\bar{S}_{2}+\bar{S}_{3}+\bar{S}_{4},
\end{aligned}
$$

where, since $a\left(P^{d} u_{h}^{n}-u_{h}^{n}, w_{d}^{n}\right)=0$,

$$
\begin{aligned}
\bar{S}_{1}= & \left(P^{d} u_{h}^{n}-u_{h}^{n}, w_{d}^{n}\right)-k b\left(\rho^{d} p_{h}^{n}-p_{h}^{n}, w_{d}^{n}\right), \\
\bar{S}_{2}= & k\left[a_{1}\left(u_{d}^{n}, P^{d} u_{h}^{n}, w_{d}^{n}\right)-a_{1}\left(u_{h}^{n}, u_{h}^{n}, w_{d}^{n}\right)+b\left(r_{d}^{n}, P^{d} u_{h}^{n}-u_{h}^{n}\right)\right], \\
\bar{S}_{3}= & \sum_{K \in \Im_{h}} \delta_{K}\left(P^{d} u_{h}^{n}-u_{h}^{n}-k \nu \Delta\left(P^{d} u_{h}^{n}-u_{h}^{n}\right)+k u_{d}^{n} \nabla P^{d} u_{h}^{n}-k u_{h}^{n} \nabla u_{h}^{n}\right. \\
& \left.+k \nabla\left(\rho^{d} p_{h}^{n}-p_{h}^{n}\right), w_{d}^{n}-k \nu \Delta w_{d}^{n}+k u_{d}^{n} \nabla w_{d}^{n}+k \nabla r_{d}^{n}\right)_{K}, \\
\bar{S}_{4}= & \left(u_{h}^{n-1}-u_{d}^{n-1}, w_{d}^{n}\right)+k \gamma j\left(T_{h}^{n}-T_{d}^{n}, w_{d}^{n}\right)+\sum_{K \in \Im_{h}} \delta_{K}\left(u_{h}^{n-1}-u_{d}^{n-1}\right. \\
& \left.+k \gamma j\left(T_{h}^{n}-T_{d}^{n}\right), w_{d}^{n}-k \nu \Delta w_{d}^{n}+k u_{d}^{n} \nabla w_{d}^{n}+k \nabla r_{d}^{n}\right)_{K} .
\end{aligned}
$$


Using inverse inequality, Hölder inequality, (2.7), and Cauchy inequality, and noting that $h=$ $O(k)$, we obtain that

$$
\begin{aligned}
& \left|\bar{S}_{1}\right|=\left|\left(P^{d} u_{h}^{n}-u_{h}^{n}, w_{d}^{n}\right)-k b\left(\rho^{d} p_{h}^{n}-p_{h}^{n}, w_{d}^{n}\right)\right| \\
& \leq C k\left(\left|P^{d} u_{h}^{n}-u_{h}^{n}\right|_{1}^{2}+\left\|\rho^{d} p_{h}^{n}-p_{h}^{n}\right\|_{0}^{2}\right)+\tilde{\varepsilon} k \nu\left|w_{d}^{n}\right|_{1}^{2}, \\
& \left|\bar{S}_{2}\right|=k\left|a_{1}\left(u_{d}^{n}, P^{d} u_{h}^{n}, w_{d}^{n}\right)-a_{1}\left(u_{h}^{n}, u_{h}^{n}, w_{d}^{n}\right)+b\left(r_{d}^{n}, P^{d} u_{h}^{n}-u_{h}^{n}\right)\right| \\
& =k \mid a_{1}\left(u_{d}^{n}, P^{d} u_{h}^{n}-u_{h}^{n}, w_{d}^{n}\right)-a_{1}\left(w_{d}^{n}, u_{h}^{n}, w_{d}^{n}\right)+a_{1}\left(P^{d} u_{h}^{n}-u_{h}^{n}, u_{d}^{n}, w_{d}^{n}\right) \\
& -\sum_{K \in \Im_{h}}\left(w_{d}^{n}-k \nu \Delta w_{d}^{n}+k u_{d}^{n} \cdot w_{d}^{n}+\nabla r_{d}^{n}, P^{d} u_{h}^{n}-u_{h}^{n}\right)_{K} \\
& +\sum_{K \in \Im_{h}}\left(w_{d}^{n}-k \nu \Delta w_{d}^{n}+k u_{d}^{n} \cdot w_{d}^{n}, P^{d} u_{h}^{n}-u_{h}^{n}\right)_{K} \mid \\
& \leq \tilde{\varepsilon}\left(k \nu\left|w_{d}^{n}\right|_{1}^{2}+\left\|\delta^{\frac{1}{2}}\left(w_{d}^{n}-k \nu \Delta w_{d}^{n}+k u_{d}^{n} \cdot w_{d}^{n}+k \nabla r_{d}^{n}\right)\right\|_{0, h}^{2}\right) \\
& +C k\left|P^{d} u_{h}^{n}-u_{h}^{n}\right|_{1}^{2}+C k h\left|w_{d}^{n}\right|_{1}^{2}, \\
& \left|\bar{S}_{3}\right|=\sum_{K \in \Im_{h}} \delta_{K}\left(P^{d} u_{h}^{n}-u_{h}^{n}-k \nu \Delta\left(P^{d} u_{h}^{n}-u_{h}^{n}\right)+k u_{d}^{n} \nabla\left(P^{d} u_{h}^{n}-u_{h}^{n}\right)\right. \\
& -k w_{d}^{n} \nabla u_{h}^{n}+k\left(P^{d} u_{h}^{n}-u_{h}^{n}\right) \nabla u_{h}^{n}+k \nabla\left(\rho^{d} p_{h}^{n}-p_{h}^{n}\right), \\
& \left.w_{d}^{n}-k \nu \Delta w_{d}^{n}+k u_{d}^{n} \nabla w_{d}^{n}+k \nabla r_{d}^{n}\right)_{K} \\
& \leq C k\left(\left|P^{d} u_{h}^{n}-u_{h}^{n}\right|_{1}^{2}+\left\|\rho^{d} p_{h}^{n}-p_{h}^{n}\right\|_{0}^{2}\right)+C k h\left|w_{d}^{n}\right|_{1}^{2} \\
& +\tilde{\varepsilon}\left\|\delta^{\frac{1}{2}}\left(w_{d}^{n}-k \nu \Delta w_{d}^{n}+k u_{d}^{n} \nabla w_{d}^{n}+k \nabla r_{d}^{n}\right)\right\|_{0, h}^{2}, \\
& \left|\bar{S}_{4}\right| \leq C k\left|P^{d} u_{h}^{n-1}-u_{h}^{n-1}\right|_{1}^{2}+\frac{1}{2}\left(\left\|w_{d}^{n-1}\right\|_{0}^{2}+\left\|w_{d}^{n}\right\|_{0}^{2}\right)+C k\left\|w_{d}^{n-1}\right\|_{0}^{2} \\
& +\tilde{\varepsilon}\left\|\delta^{\frac{1}{2}}\left(w_{d}^{n}-k \nu \Delta w_{d}^{n}+k u_{d}^{n} \nabla w_{d}^{n}+k \nabla r_{d}^{n}\right)\right\|_{0, h}^{2} \\
& +\tilde{\varepsilon} k \nu\left|w_{d}^{n}\right|_{1}+C k^{3}\left\|T_{h}^{n}-T_{d}^{n}\right\|_{0}^{2},
\end{aligned}
$$

where $\tilde{\varepsilon}$ is a constant which can be chosen arbitrarily. Combining (4.33) and (4.34)-(4.38) could yield that

$$
\begin{aligned}
& \left\|w_{d}^{n}\right\|_{0}^{2}+k \nu\left|w_{d}^{n}\right|_{1}^{2}+\left\|\delta^{\frac{1}{2}}\left(w_{d}^{n}-k \nu \Delta w_{d}^{n}+k u_{d}^{n} \nabla w_{d}^{n}+k \nabla r_{d}^{n}\right)\right\|_{0, h}^{2} \\
\leq & C k\left(\left|P^{d} u_{h}^{n}-u_{h}^{n}\right|_{1}^{2}+\left\|\rho^{d} p_{h}^{n}-p_{h}^{n}\right\|_{0}^{2}+\left|P^{d} u_{h}^{n-1}-u_{h}^{n-1}\right|_{1}^{2}\right) \\
& +3 \tilde{\varepsilon} k\left(\nu\left|w_{d}^{n}\right|_{1}^{2}+\left\|\delta^{\frac{1}{2}}\left(w_{d}^{n}-k \nu \Delta w_{d}^{n}+k u_{d}^{n} \nabla w_{d}^{n}+k \nabla r_{d}^{n}\right)\right\|_{0, h}^{2}\right) \\
& +C k h\left|w_{d}^{n}\right|_{1}^{2}+\frac{1}{2}\left(\left\|w_{d}^{n-1}\right\|_{0}^{2}+\left\|w_{d}^{n}\right\|_{0}^{2}\right)+C k\left\|w_{d}^{n-1}\right\|_{0}^{2}+C k^{3}\left\|T_{h}^{n}-T_{d}^{n}\right\|_{0}^{2} .
\end{aligned}
$$


Taking $\tilde{\varepsilon} \leq 1 / 6$, from (4.39) we obtain that

$$
\begin{aligned}
& \left\|w_{d}^{n}\right\|_{0}^{2}+k \nu\left|w_{d}^{n}\right|_{1}^{2}+\left\|\delta^{\frac{1}{2}}\left(w_{d}^{n}-k \nu \Delta w_{d}^{n}+k u_{d}^{n} \nabla w_{d}^{n}+k \nabla r_{d}^{n}\right)\right\|_{0, h}^{2} \\
\leq \quad & C k\left(\left|P^{d} u_{h}^{n}-u_{h}^{n}\right|_{1}^{2}+\left\|\rho^{d} p_{h}^{n}-p_{h}^{n}\right\|_{0}^{2}+\left|P^{d} u_{h}^{n-1}-u_{h}^{n-1}\right|_{1}^{2}\right) \\
& +C k h\left|w_{d}^{n}\right|_{1}^{2}+\left\|w_{d}^{n-1}\right\|_{0}^{2}+C k\left\|w_{d}^{n-1}\right\|_{0}^{2}+C k^{3}\left\|T_{h}^{n}-T_{d}^{n}\right\|_{0}^{2} .
\end{aligned}
$$

If $h$ is sufficiently small such that $C h \leq \nu / 2$, we could obtain from above inequality that

$$
\begin{aligned}
& 2\left\|w_{d}^{n}\right\|_{0}^{2}+k \nu\left|w_{d}^{n}\right|_{1}^{2}+2\left\|\delta^{\frac{1}{2}}\left(w_{d}^{n}-k \nu \Delta w_{d}^{n}+k u_{d}^{n} \nabla w_{d}^{n}+k \nabla r_{d}^{n}\right)\right\|_{0, h}^{2} \\
\leq & C k\left(\left|P^{d} u_{h}^{n}-u_{h}^{n}\right|_{1}^{2}+\left\|\rho^{d} p_{h}^{n}-p_{h}^{n}\right\|_{0}^{2}+\left|P^{d} u_{h}^{n-1}-u_{h}^{n-1}\right|_{1}^{2}\right) \\
& +2\left\|w_{d}^{n-1}\right\|_{0}^{2}+C k\left\|w_{d}^{n-1}\right\|_{0}^{2}+C k^{3}\left\|T_{h}^{n}-T_{d}^{n}\right\|_{0}^{2} .
\end{aligned}
$$

Let $\tau_{d}^{n}=\varrho^{d} T_{h}^{n}-T_{d}^{n}$. By (2.6), (4.11), (4.12), and inverse inequality, we could get that

$$
\begin{aligned}
& \left\|\tau_{d}^{n}\right\|_{0}^{2}+k \gamma^{-1}\left|\tau_{d}^{n}\right|_{1}^{2}=\left(\varrho^{d} T_{h}^{n}-T_{h}^{n}, \tau_{d}^{n}\right)+\left(\tau_{d}^{n-1}, \tau_{d}^{n}\right) \\
& -k a_{2}\left(u_{h}^{n}-u_{d}^{n}, T_{h}^{n}, \tau_{d}^{n}\right)+k a_{2}\left(u_{d}^{n}, \varrho^{d} T_{h}^{n}-T_{h}^{n}, \tau_{d}^{n}\right)+\left(T_{h}^{n-1}-\varrho^{d} T_{h}^{n-1}, \tau_{d}^{n}\right) \\
& \leq \frac{k \gamma^{-1}}{2}\left|\tau_{d}^{n}\right|_{1}^{2}+\frac{1}{2}\left\|\tau_{d}^{n}\right\|_{0}^{2}+\frac{1}{2}\left\|\tau_{d}^{n-1}\right\|_{0}^{2}+C k\left|u^{n}-u_{d}^{n}\right|_{1}^{2} \\
& +C k\left\|T_{h}^{n-1}-\varrho^{d} T_{h}^{n-1}\right\|_{0}^{2}+C k\left|T_{h}^{n}-\varrho^{d} T_{h}^{n}\right|_{1}^{2} .
\end{aligned}
$$

Therefore, we have

$$
\begin{aligned}
& \left\|\tau_{d}^{n}\right\|_{0}^{2}+k \gamma^{-1}\left|\tau_{d}^{n}\right|_{1}^{2} \leq\left\|\tau_{d}^{n-1}\right\|_{0}^{2} \\
& +C k\left\|T_{h}^{n-1}-\varrho^{d} T_{h}^{n-1}\right\|_{0}^{2}+C k\left|T_{h}^{n}-\varrho^{d} T_{h}^{n}\right|_{1}^{2}+C k\left|u^{n}-u_{d}^{n}\right|_{1}^{2} .
\end{aligned}
$$

First, we consider the case of $n \in\{1,2, \cdots, L\}$. Summing (4.43) from 1 to $n \in\{1,2, \cdots, L\}$ and using Lemma 3.2 could yield that

$$
\left\|\tau_{d}^{n}\right\|_{0}^{2}+k \gamma^{-1} \sum_{i=1}^{n}\left|\tau_{d}^{i}\right|_{1}^{2} \leq C k \sum_{i=1}^{n}\left|u^{i}-u_{d}^{i}\right|_{1}^{2}+C k L^{2}\left(\sum_{i=d+1}^{l} \lambda_{i}\right)^{2} .
$$

Thus, if $k=O\left(L^{-2}\right)$,

$$
\left\|T^{n}-T_{d}^{n}\right\|_{0}^{2}+k \gamma^{-1} \sum_{i=1}^{n}\left|T^{n}-T_{d}^{n}\right|_{1}^{2} \leq C k \sum_{i=1}^{n}\left|u^{i}-u_{d}^{i}\right|_{1}^{2}+C\left(\sum_{i=d+1}^{l} \lambda_{i}\right)^{2} .
$$

Summing (4.41) from 1 to $n \in\{1,2, \cdots, L\}$ and using Lemma 3.2 yields that

$$
\begin{aligned}
& \left\|w_{d}^{n}\right\|_{0}^{2}+k \nu \sum_{i=1}^{n}\left|w_{d}^{i}\right|_{1}^{2}+\sum_{i=1}^{n}\left\|\delta^{\frac{1}{2}}\left(w_{d}^{i}-k \nu \Delta w_{d}^{i}+k u_{d}^{i} \nabla w_{d}^{i}+k \nabla r_{d}^{i}\right)\right\|_{0, h}^{2} \\
& \leq C k L^{2}\left(\sum_{j=d+1}^{l} \lambda_{j}\right)^{2}+C k \sum_{i=0}^{n-1}\left\|w_{d}^{i}\right\|_{0}^{2}+C k^{3} \sum_{i=1}^{n}\left\|T^{i}-T_{d}^{i}\right\|_{0}^{2} .
\end{aligned}
$$


By using discrete Gronwall inequality, we obtain that

$$
\begin{aligned}
& \left\|w_{d}^{L}\right\|_{0}^{2}+k \nu \sum_{i=1}^{n}\left|w_{d}^{i}\right|_{1}^{2}+\sum_{i=1}^{n}\left\|\delta^{\frac{1}{2}}\left(w_{d}^{i}-k \nu \Delta w_{d}^{i}+k u_{d}^{i} \nabla w_{d}^{i}+k \nabla r_{d}^{i}\right)\right\|_{0, h}^{2} \\
& \leq C k\left[L^{2}\left(\sum_{j=d+1}^{l} \lambda_{j}\right)^{2}+k^{2} \sum_{i=1}^{n}\left\|T^{i}-T_{d}^{i}\right\|_{0}^{2}\right] \exp (C k n) .
\end{aligned}
$$

If $h$ and $k$ are sufficiently small, $k=O\left(L^{-2}\right)$, by using inverse inequality and noting that $n k \leq k N \leq T$, we get that

$$
\left\|w_{d}^{n}\right\|_{0}+(k \nu)^{\frac{1}{2}} \sum_{i=1}^{n}\left|w_{d}^{i}\right|_{1}+k^{\frac{1}{2}} \sum_{i=1}^{n}\left\|r_{d}^{i}\right\|_{0} \leq C \sum_{j=d+1}^{l} \lambda_{j}+C\left[k^{3} \sum_{i=1}^{n}\left\|T^{i}-T_{d}^{i}\right\|_{0}^{2}\right]^{1 / 2} .
$$

Using Lemma 3.2 and (4.45) yields that

$$
\begin{aligned}
& \left\|u_{h}^{n}-u_{d}^{n}\right\|_{0}+(k \nu)^{\frac{1}{2}} \sum_{i=1}^{n}\left|u_{h}^{i}-u_{d}^{i}\right|_{1}+k^{\frac{1}{2}} \sum_{i=1}^{n}\left\|p_{h}^{i}-p_{d}^{i}\right\|_{0} \\
& \leq C \sum_{j=d+1}^{l} \lambda_{j}+C k^{3 / 2} \sum_{i=1}^{n}\left|u_{h}^{i}-u_{d}^{i}\right|_{1} .
\end{aligned}
$$

If $k$ are sufficiently small, for example, $C k \leq(\nu)^{\frac{1}{2}} / 2$, by $(4.49)$ we obtain that

$$
\left\|u_{h}^{n}-u_{d}^{n}\right\|_{0}+(k \nu)^{\frac{1}{2}} \sum_{i=1}^{n}\left|u_{h}^{i}-u_{d}^{i}\right|_{1}+k^{\frac{1}{2}} \sum_{i=1}^{n}\left\|p_{h}^{i}-p_{d}^{i}\right\|_{0} \leq C \sum_{j=d+1}^{l} \lambda_{j} .
$$

Combining (4.50) and (4.45) can yield (4.31).

Next, we consider the case of $n \notin\{1,2, \cdots, L\}$. If $n \notin\{1,2, \cdots, L\}$, we may as well suppose that $t_{n} \in\left(t_{L-1}, t_{L}\right)$ and $t_{n}$ be the nearest point to $t_{L}$. Expanding $u_{h}^{n}, p_{h}^{n}$, and $T_{h}^{n}$ into Taylor series with respect to $t_{L}$ could yield that

$$
\begin{array}{ll}
u_{h}^{n}=u_{h}^{L}-\eta k \frac{\partial u_{h}\left(\xi_{1}\right)}{\partial t} & \xi_{1} \in\left[t_{n}, t_{L}\right], \\
p_{h}^{n}=p_{h}^{L}-\eta k \frac{\partial p_{h}\left(\xi_{2}\right)}{\partial t} & \xi_{2} \in\left[t_{n}, t_{L}\right], \\
T_{h}^{n}=T_{h}^{L}-\eta k \frac{\partial T_{h}\left(\xi_{3}\right)}{\partial t} & \xi_{3} \in\left[t_{n}, t_{L}\right],
\end{array}
$$

where $\eta$ is the step number from $t_{n}$ to $t_{L}$. If snapshots are equably taken, then $\eta \leq N /(2 L)$. Summing (4.43) and (4.41) from 1 to $L-1, n$, and using (4.51), if $\left|\frac{\partial u_{h}\left(\xi_{1}\right)}{\partial t}\right|,\left|\frac{\partial p_{h}\left(\xi_{2}\right)}{\partial t}\right|$, and $\left|\frac{\partial T_{h}\left(\xi_{3}\right)}{\partial t}\right|$ are bounded, by discrete Gronwall inequality and Lemma 3.2, we obtain that

$$
\begin{aligned}
\left\|\tau_{d}^{n}\right\|_{0}^{2}+k \gamma^{-1} & \sum_{i=1}^{n}\left|\tau_{d}^{i}\right|_{1}^{2} \leq C k \sum_{i=1}^{n}\left|u^{i}-u_{d}^{i}\right|_{1}^{2}+C k\left(L \sum_{j=a+1}^{l} \lambda_{j}\right)^{2}+C L k^{3} N^{2} /(2 L)^{2} . \\
& \left\|w_{d}^{n}\right\|_{0}^{2}+k \nu \sum_{i=1}^{n}\left|w_{d}^{i}\right|_{1}^{2}+k \sum_{i=1}^{n}\left\|r_{d}^{i}\right\|_{0}^{2} \\
& \leq C k\left(L \sum_{j=a+1}^{l} \lambda_{j}\right)^{2}+C L k^{3} N^{2} /(2 L)^{2}+C k^{3} \sum_{i=1}^{n}\left\|T^{i}-T_{d}^{i}\right\|_{0}^{2} .
\end{aligned}
$$


If $k=O\left(L^{-2}\right)$, by (4.52) and (4.53) we get that

$$
\begin{gathered}
\left\|\tau_{d}^{n}\right\|_{0}+k \gamma^{-1} \sum_{i=1}^{n}\left|\tau_{d}^{i}\right|_{1} \leq C k^{1 / 2}\left[\sum_{i=1}^{n}\left|w_{d}^{i}\right|_{1}^{2}\right]^{1 / 2}+C \sum_{j=a+1}^{l} \lambda_{j}+C k \sqrt{\frac{N}{4 L}} \\
\left\|w_{d}^{n}\right\|_{0}+(k \nu)^{\frac{1}{2}} \sum_{i=1}^{n}\left|w_{d}^{i}\right|_{1}+k^{\frac{1}{2}} \sum_{i=1}^{n}\left\|r_{d}^{i}\right\|_{0} \\
\leq C \sum_{j=a+1}^{l} \lambda_{j}+C k \sqrt{\frac{N}{4 L}}+C k^{3 / 2}\left[\sum_{i=1}^{n}\left\|\tau_{d}^{i}\right\|_{0}^{2}\right]^{1 / 2} .
\end{gathered}
$$

Combining (4.54) and (4.55), by Lemma 3.2, we obtain (4.32).

Combining Theorem 2.4 and Theorem 4.3 yields the following result.

THEOREM 4.4. Under Theorem 2.4 and Theorem 4.3 hypotheses, the error estimates between the solutions $(u(t), p(t), T(t))$ for Problem $(\mathrm{I})$ and the solutions $\left(u_{d}^{n}, p_{d}^{n}, T_{d}^{n}\right)$ for the reduced order basic Problem (V) are, for $n=1,2, \cdots, N$,

$$
\begin{aligned}
& \left\|u\left(t_{n}\right)-u_{d}^{n}\right\|_{0}+\left\|T\left(t_{n}\right)-T_{d}^{n}\right\|_{0}+(k \nu)^{\frac{1}{2}} \sum_{i=1}^{n}\left\|\nabla\left(u^{i}-u_{d}^{i}\right)\right\|_{0}+k^{\frac{1}{2}} \sum_{i=1}^{n}\left\|p^{i}-p_{d}^{i}\right\|_{0} \\
& +\left(k \gamma^{-1}\right)^{\frac{1}{2}} \sum_{i=1}^{n}\left\|\nabla\left(T^{i}-T_{d}^{i}\right)\right\|_{0} \leq C\left(h^{\kappa}+h^{\ell}+h^{\iota}+k\right)+C \sum_{j=d+1}^{l} \lambda_{j} \text {, if } n \in\{1,2, \cdots, L\} ; \\
& \quad\left\|u\left(t_{n}\right)-u_{d}^{n}\right\|_{0}+\left\|T\left(t_{n}\right)-T_{d}^{n}\right\|_{0}+(k \nu)^{\frac{1}{2}} \sum_{i=1}^{n}\left\|\nabla\left(u^{i}-u_{d}^{i}\right)\right\|_{0}+k^{\frac{1}{2}} \sum_{i=1}^{n}\left\|p^{i}-p_{d}^{i}\right\|_{0} \\
& \quad+\left(k \gamma^{-1}\right)^{\frac{1}{2}} \sum_{i=1}^{n}\left\|\nabla\left(T^{i}-T_{d}^{i}\right)\right\|_{0} \leq C\left(h^{\kappa}+h^{\ell}+h^{\iota}+k+k \sqrt{\frac{N}{4 L}}\right) \\
& \quad+C \sum_{j=d+1}^{l} \lambda_{j}, \text { if } n \notin\{1,2, \cdots, L\} .
\end{aligned}
$$

Remark 2. The conditions $k=O\left(L^{-2}\right)$ and the coefficient $\sqrt{\frac{N}{4 L}}$ in Theorem 4.3 and Theorem 4.4 show that enough snapshots must be taken, but if only $\sqrt{\frac{N}{4 L}}<10$, then error estimates are almost optimal. For example, if only one snapshot is taken from each 10 transient solutions, error estimates could be satisfactory. Therefore, it is unnecessary to take the total transient solutions at all time instances $t_{n}$ as snapshot for instance in [30-31]. Theorem 4.3 and Theorem 4.4 have presented the error estimates between the solutions of the optimizing reduced PLSMFE formulation Problem (V) and the solutions of usual PLSMFE formulation Problem (III) and Problem (II), respectively. Since our methods employ some usual PLSMFE solutions $\left(u_{h}^{n}, p_{h}^{n}, T_{h}^{n}\right)(n=1,2, \cdots, L)$ for Problem (III) as assistant analysis, the error estimates in Theorem 4.4 are correlated to the spatial grid scale $h$ and time step size $k$. However, when one computes actual problems, one may obtain the ensemble of snapshots from physical system trajectories by drawing samples from experiments and interpolation (or data assimilation). For example, for weather forecast, one can use previous weather prediction results to construct the ensemble of snapshots. Thus, the assistant analysis $\left(u_{h}^{n}, p_{h}^{n}, T_{h}^{n}\right)(n=1,2, \cdots, L)$ could 
be substituted by the interpolation functions of experimental and previous results, it is not necessary to solve Problem (III), and it is only necessary to directly solve Problem (V) which includes very few degrees of freedom since it is only dependent on $d$ and is independent of the spatial grid scale $h$ and time step size $k$, and, in general, $d(d \ll l \leq L \ll N)$.

\section{Some numerical experiments}

In this section, we present some numerical examples of the physical model of cavity flows for first order element (i.e., $\ell=\kappa=\iota=1$ ) and different Reynolds numbers by the optimizing reduced PLSMFE formulation Problem (V) validating the feasibility and efficiency of the POD method.

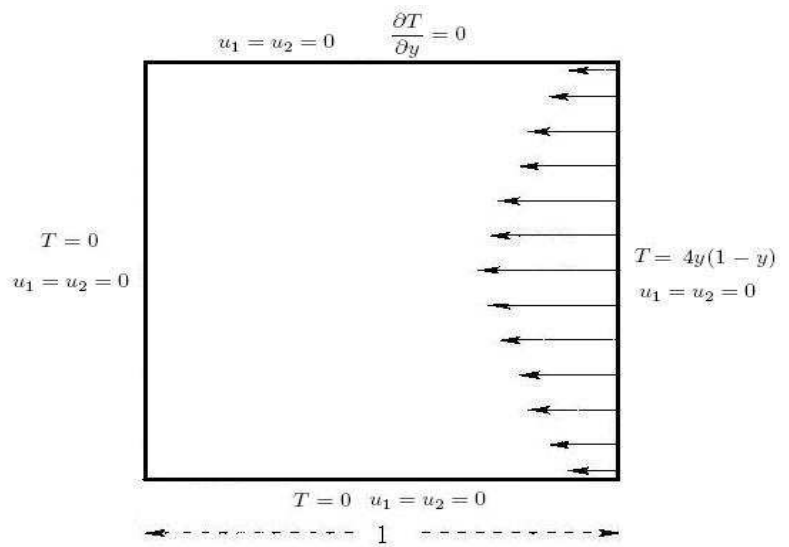

Figure 1. Physics model of the cavity flows: $t=0$ i.e., $n=0$ initial values on boundary
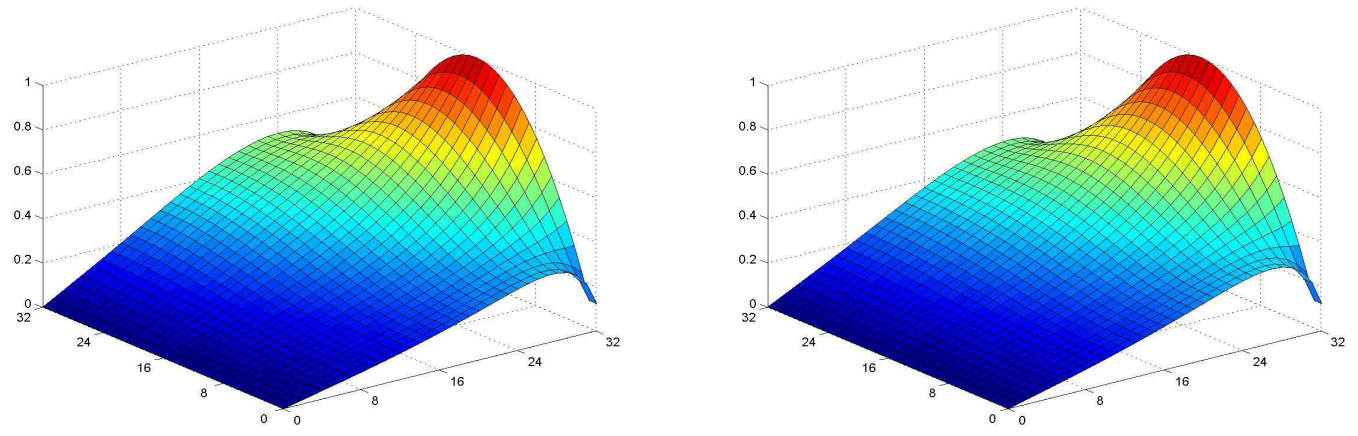

Figure 2. When $\operatorname{Re}=2000$, temperature figure for classical PLSMFE solution (on left-hand side figure) and when $d=5$ the optimizing reduced PLSMFE solution based on POD (on right-hand side figure)

Let the side length of the cavity be 1 (see Figure 1 ). We first divide the cavity into $32 \times 32=$ 1024 small squares with side length $\triangle x=\triangle y=\frac{1}{32}$, and then link diagonal of square to divide each square into two triangles on same direction which consists of triangularization $\Im_{h}\left(h=\frac{\sqrt{2}}{32}\right)$. We take time step increment as $\triangle t=0.001$. Let the initial value and boundary value of $u$ and $v$ equal to 0 on boundary of the cavity are also taken as 0 . And let $T=0$ on left and lower 
boundary of cavity, $\frac{\partial T}{\partial y}=0$ on supper boundary of cavity, and $T=4 y(1-y)$ on right boundary of cavity (see Figure 1). Put $\gamma=10$ and $R e=2000$ or 5000.
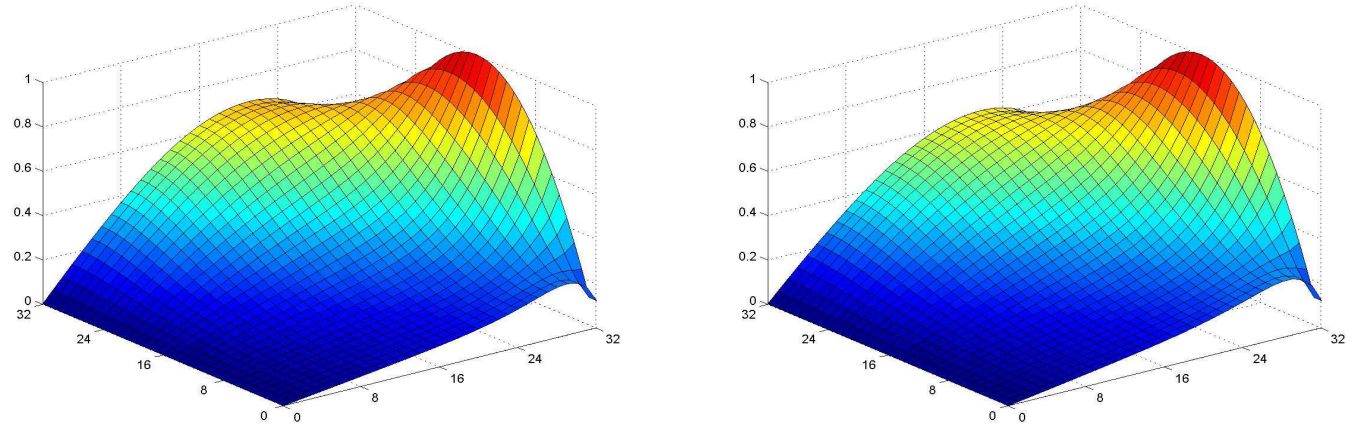

Figure 3. When $\operatorname{Re}=5000$, temperature figure for classical PLSMFE solution (on left-hand side figure) and when $d=5$ the optimizing reduced PLSMFE solution based on POD (on right-hand side figure)
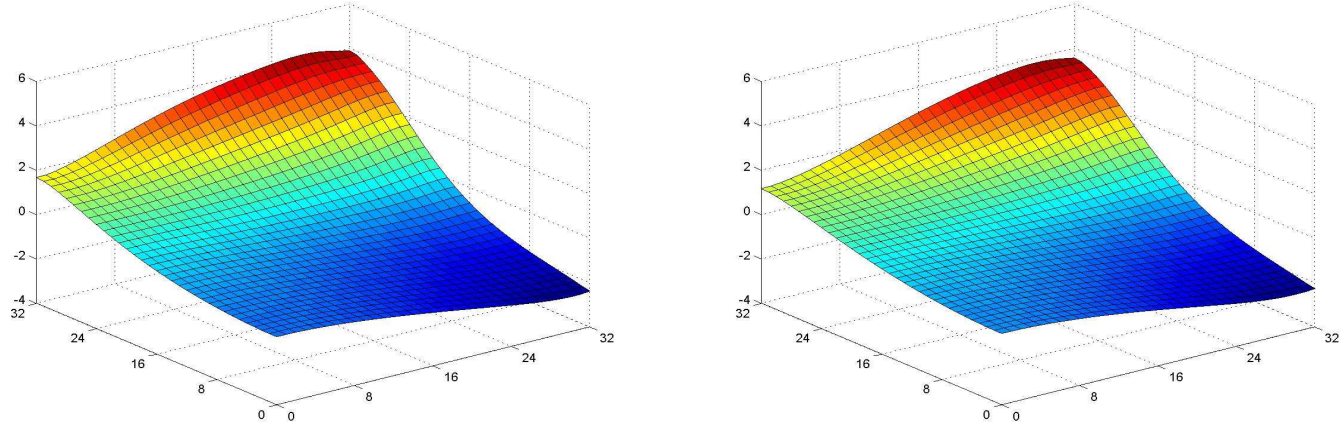

Figure 4. When $\mathrm{Re}=2000$, pressure Figure for classical PLSMFE solution (on left-hand side figure) and when $d=5$ the optimizing reduced PLSMFE solution based on POD (on right-hand side Figure)
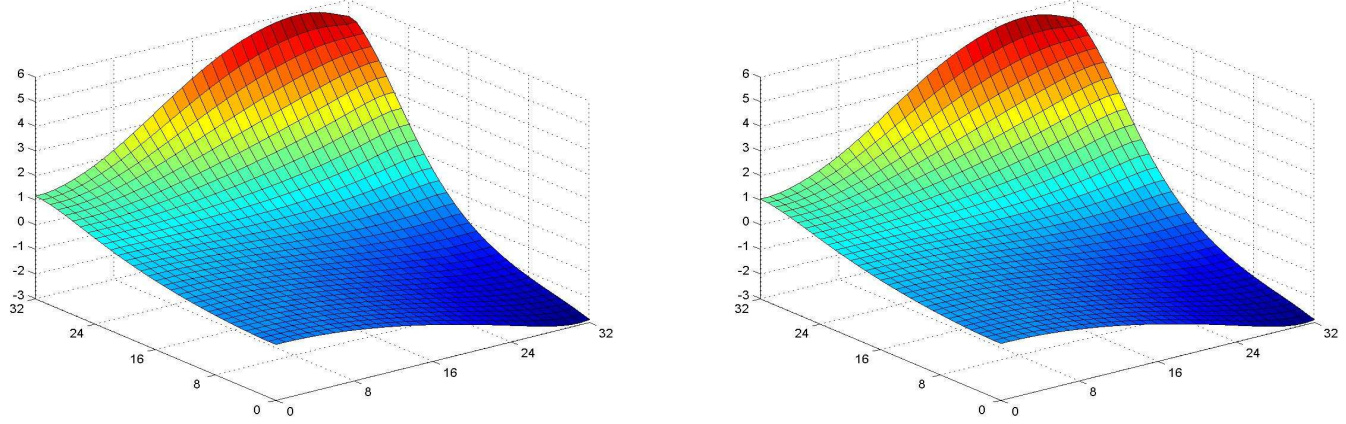

Figure 5. When $R e=5000$, pressure stream line figure for classical PLSMFE solutions (on left-hand side Figure) and when $d=5$ the optimizing reduced PLSMFE solution based on POD (on right-hand side Figure)

We obtain 20 values (i.e., snapshots) outputting at time $t=10,20,30, \cdots, 200$ by solving classical PLSMFE formulation, i.e., Problem (IV). It is shown by computing that the eigenvalues $\sum_{i=6}^{20} \lambda_{i} \leq 3 \times 10^{-3}$. When $t=200$, we obtain the solutions of the reduced formulation Problem 
(IV) based POD method of MEF depicted graphically in Figure 2 to Figure 7 on right-hand side used 5 optimal POD bases if $R e=2000$ and $R e=5000$, while the solutions obtained with classical PLSMFE formulation Problem (III) are depicted graphically in Figure 2 to Figure 7 on left-hand side (Since these figures are equal to solutions obtained with 20 bases, they are also known as the figures of solution with full bases).
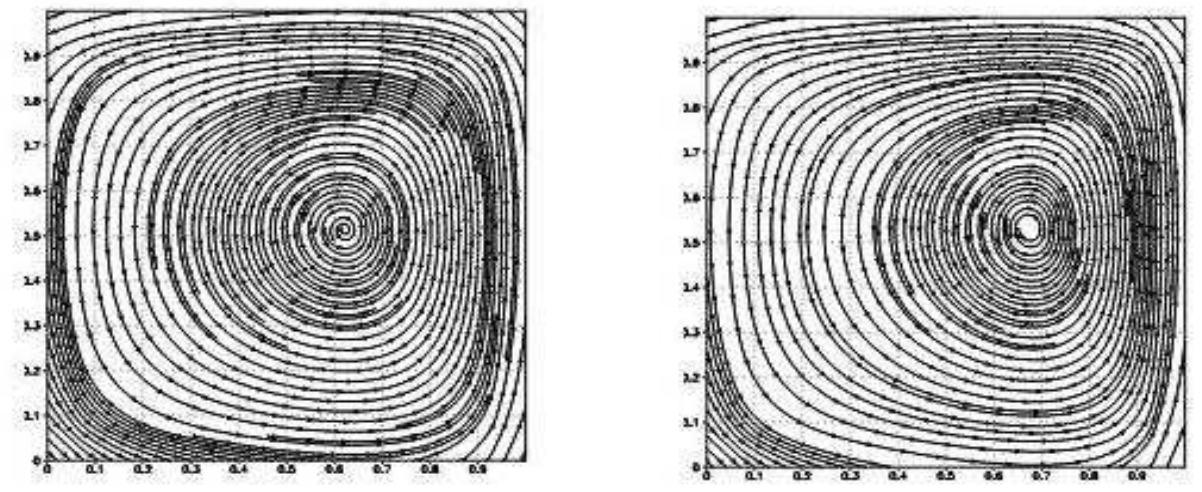

Figure 6. When Re=2000, velocity stream line figure for classical PLSMFE solutions (on left-hand side Figure) and when $d=5$ the optimizing reduced PLSMFE solution based on POD (on right-hand side Figure)
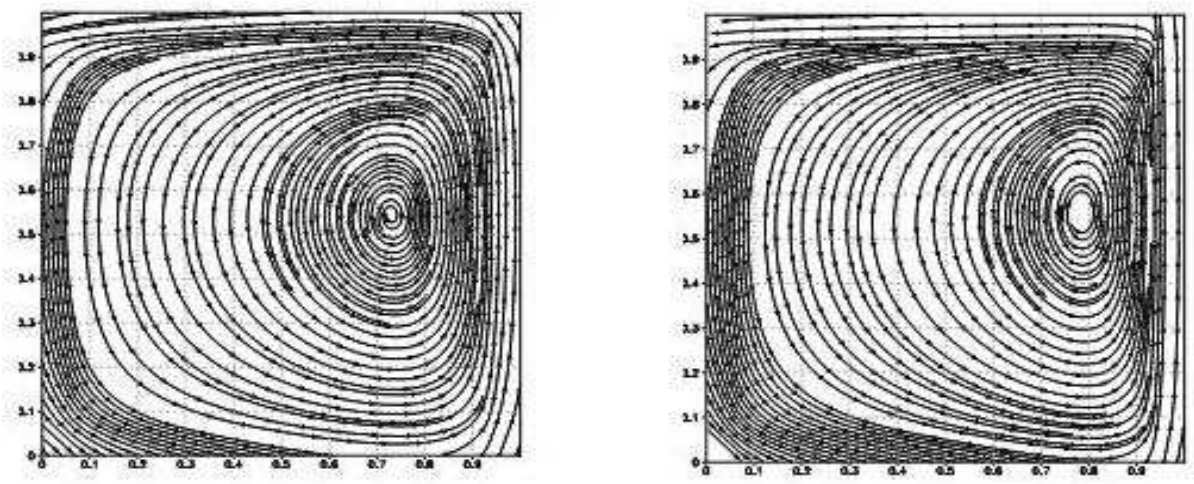

Figure 7. When $\mathrm{Re}=5000$, velocity stream line figure for classical PLSMFE solutions (on left-hand side Figure) and when $d=5$ the optimizing of the reduced PLSMFE solution based on POD (on right-hand side Figure)

Figure 8 shows the errors between solutions obtained with different number of optimal POD bases and solutions obtained with full bases. Comparing classical PLSMFE formulation Problem (III) with the reduced PLSMFE formulation Problem (IV) containing five optimal bases implementing 3000 times numerical simulation computations, we find that classical for PLSMFE formulation Problem (III) CPU time required is five minutes, while the optimizing reduced PLSMFE formulation Problem (IV) with five optimal bases required CPU time is only three seconds, i.e., classical PLSMFE formulation Problem (III) required CPU time is by a factor of 120 larger than the optimizing reduced PLSMFE formulation Problem (IV) 
with five optimal bases required CPU time, while the errors between their solutions do not exceed $4 \times 10^{-3}$. It is also shown that finding the approximate solutions for the non-stationary conduction-convection problems with the optimizing reduced PLSMFE formulation Problem (IV) is very effective. And the results obtained for numerical examples are consistent with theoretical those.

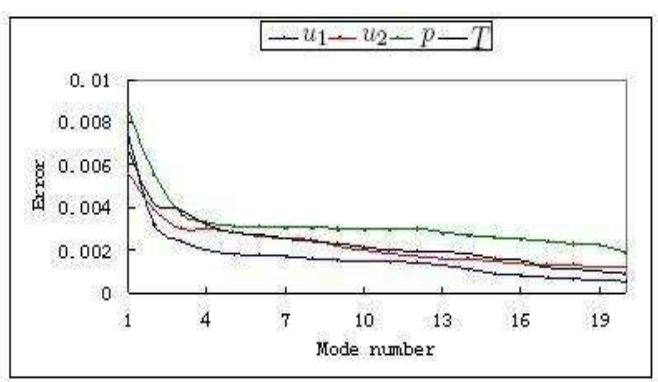

$\mathrm{Re}=2000$

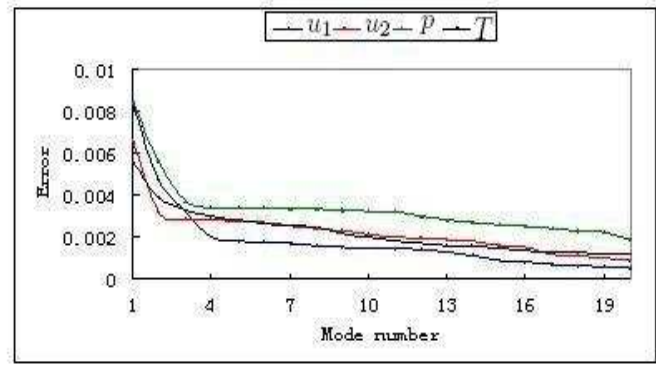

$\operatorname{Re}=5000$

Figure 8. Error for $\mathrm{Re}=2000$ on left-hand side, error for $\mathrm{Re}=5000$ on right-hand side

\section{Conclusions}

In this paper, we have employed the POD techniques to derive an optimizing reduced PLSMFE formulation for the non-stationary conduction-convection problems. We first reconstruct optimal orthogonal bases of ensembles of data which are compiled from transient solutions derived by using usual PLSMFE equation system, while in actual applications, one may obtain the ensemble of snapshots from physical system trajectories by drawing samples from experiments and interpolation (or data assimilation). For example, for weather forecast, one can use previous weather prediction results to construct the ensemble of snapshots to restructure the POD basis for the ensemble of snapshots by methods of above section 3. We have also combined the optimal orthogonal bases with a Petrov-Galerkin least squares projection procedure, thus yielding a new optimizing reduced PLSMFE formulation of lower dimensional order and of high accuracy for the non-stationary conduction-convection problems. We have then proceeded to derive error estimates between our optimizing reduced PLSMFE approximate solutions and the usual PLSMFE approximate solutions, and have shown using numerical examples that the errors between the optimizing reduced PLSMFE approximate solutions and the usual PLSMFE solutions are consistent with the theoretical error results, thus validating both feasibility and efficiency of our optimizing reduced PLSMFE formulation. Future research work in this area will aim to extend the optimizing reduced PLSMFE formulation, applying it to a realistic atmosphere quality forecast system and to more complicated PDEs. From theoretical analysis and numerical examples, we have shown that the optimizing reduced PLSMFE formulation presented herein has extensive perspective applications.

Though Kunisch and Volkwein have presented some Galerkin proper orthogonal decompo- 
sition methods for a general equation in fluid dynamics, i.e., for the non-stationary conductionconvection problems in [31], our method is different from their approaches, whose methods consist of Galerkin projection approaches where original variables are substituted for linear combination of POD basis and the error estimates of the velocity field therein are only derived, their POD basis is generated with the solutions of the physical system at all time instances, while our POD basis is generated with few solutions of the physical system which are useful and of interest for us. In particular,the velocity field is only approximated in Reference [31], while velocity field and pressure are all synchronously approximated in our method, and the error estimates of both velocity field and pressure approximate solutions are also synchronously derived. Thus our method appears to be more optimal than that in [31].

\section{REFERENCES}

1. E. DiBenedetto, A. Friedman (1986): Conduction-convection problems with change of phase. J. Diff. Equ. 62: 129-185

2. P.M. Gresho, R.L. Lee, S.T. Chen (1980): Solution of the time-dependent incompressible NavierStokes and Boussinesq equation using the Galerkin finite element method. Lecture Notes in Math.

3. Luo Zhen-dong, Zhu Jiang, Xie Zheng-hui (2003): A difference scheme and numerical simulation based on mixed finite element method for natural convection problem. Applied Mathematics and Mechanics 24(9): 973-983

4. Luo Zhen-dong (1998): The mixed finite element method for the non stationary Conduction convection problems. C. J. Numer. Math. \& Appl. 20(2): 29-59

5. T.J. Hughes, L.P. France, and M. Balestra (1986): A new finite element formulation for computational fluid dynamics. V. Circumventing the Bubuška-Brezzi condition: A stable Petrov Galerkin formulation of the Stokes problem accommodating equal-order interpolation, Comput. Meth. Appl. Mech. Engrg., 59: 85-99

6. T.J. Hughes and L.P. France (1987): A new finite element formulation for computational fluid dynamics. VII.The Stokes problem with various well posed boundary conditions, symmetric formulations that converge for all velocity pressure space, Comput.Meth.Appl.Mech. Engrg., 65: $85-96$

7. F. Brezzi and J. Douglas Jr (1988): Stabilized mixed method for the Stokes problem, Numer. Math., 53: 225-235

8. J. Douglas Jr. and J.P. Wang (1989): An absolutely stabilized finite element method for the stokes problem, Math. Comp., 52: 495-508

9. T.J. Hughes and T.E. Tezduyar (1984): Finite element methods for first-order hyperbolic systems with particular emphasis on the compressible Euler equations, Comput. Meth. Appl. Mech. Engrg., 45: 217-284

10. C. Johnson and J. Saranen (1986): Streamline diffusion methods for the incompressible Euler and Navier-Stokes equations, Math. Comp., 47: 1-18

11. P. Hansbo and A. Szepessy (1990): A velocity - pressure streamline diffusion finite element method for the incompressible Navier-Stokes equations, Comput. Meth. Appl. Mech. Engrg., 84: 175-192 
12. T.X. Zhou and M.F. Feng (1993): A least squares Petrov-Galerkin finite element method for the stationary Navier - Stokes equations, Math. Comp., 60 531-543.

13. Luo Zhen-dong and Chen Jing: Petrov least squares mixed finite element method for the non stationary conduction convection problems, to submit Mathematica Numerica Sinica, (2007)

14. K. Fukunaga (1990): Introduction to Statistical Recognition. Academic Press

15. I.T. Jolliffe (2002): Principal Component Analysis. Springer-Verlag

16. P. Holmes, J.L. Lumley, G. Berkooz (1996): Turbulence, Coherent Structures, Dynamical Systems and Symmetry. Cambridge University Press, Cambridge, UK

17. J.L. Lumley (1981): Coherent Structures in Turbulence, in Meyer R E (ed.), Transition and Turbulence. Academic Press, pp. 215-242

18. N. Aubry, P. Holmes, J.L. Lumley et al. (1988): The dynamics of coherent structures in the wall region of a turbulent boundary layer. Journal of Fluid Dynamics 192: 115-173

19. L. Sirovich (1987): Turbulence and the dynamics of coherent structures: Part I-III. Quarterly of Applied Mathematics 45(3): 561-590

20. P. Moin, R.D. Moser (1989): Characteristic-eddy decomposition of turbulence in channel. Journal of Fluid Mechanics 200: 417-509

21. M. Rajaee, S.K.F. Karlsson, L. Sirovich (1994): Low dimensional description of free shear flow coherent structures and their dynamical behavior. Journal of Fluid Mechanics 258: 1401-1402

22. R.D. Roslin, M.D. Gunzburger, R. Nicolaides, et al. (1997): A self-contained automated methodology for optimal flow control validated for transition delay. AIAA Journal 35: 816-824

23. H.V. Ly, H.T. Tran (2002): Proper orthogonal decomposition for flow calculations and optimal control in a horizontal CVD reactor. Quarterly of Applied Mathematics 60: 631-656

24. J. Ko, A.J. Kurdila, O.K. Redionitis, et al. (1999): Synthetic Jets, Their Reduced Order Modeling and Applications to Flow Control. AIAA Paper number 99-1000, 37 Aerospace Sciences Meeting \& Exhibit, Reno

25. Y.H. Cao, J. Zhu, Z.D. Luo, I.M. Navon (2006): Reduced order modeling of the upper tropical pacific ocean model using proper orthogonal decomposition. Computers \& Mathematics with Applications 52: 1373-1386

26. Y.H. Cao, J. Zhu, I.M. Navon, Z.D. Luo (2007): A reduced order approach to four-dimensional variational data assimilation using proper orthogonal decomposition. International Journal for Numerical Methods in Fluids 53: 1571-1583

27. Z.D. Luo, J. Zhu, R.W. Wang, and I.M. Navon: Proper orthogonal decomposition approach and error estimation of mixed finite element methods for the tropical Pacific Ocean reduced gravity model. Computer Methods in Applied Mechanics and Engineering (2007 in print)

28. Z.D. Luo, J. Chen, J. Zhu, R.W. Wang, I.M. Navon: An optimizing reduced order FDS for the tropical Pacific Ocean reduced gravity model. International Journal for Numerical Methods in Fluids (DoI:10. 1002/fld. 1452, 2007 reprint).

29. Z.D. Luo, J. Chen, R.W. Wang: Reduced FDS based on singular value decomposition for the nonstationary conduction-convection problems. to submit Numerische Mathematik (May, 2007).

30. K. Kunisch, S. Volkwein (2001): Galerkin proper orthogonal decomposition methods for parabolic problems. Numerische Mathematik 90: 177-148

31. K. Kunisch, S. Volkwein (2002): Galerkin proper orthogonal decomposition methods for a general equation in fluid dynamics. SIAM J. Numer. Anal. 40(2): 492-515 
32. K. Kunisch, S. Volkwein (1999): Control of Burgers' equation by a reduced order approach using proper orthogonal decomposition. Journal of Optimization Theory and Applications 102: 345-371

33. D. Ahlman, F. Södelund, J. Jackson, A. Kurdila, W. Shyy (2002): Proper orthogonal decomposition for time-dependent lid-driven cavity flows. Numerical Heal Transfer Part B-Fundamentals 42(4): 285-306

34. R.A. Adams (1975): Sobolev Space. New York: Academic Press

35. Z.D. Luo (2006): Mixed Finite Element Methods and Applications. Beijing: Chinese Science Press

36. P.G. Ciarlet (1978): The Finite Element Method for Elliptic Problems. North-Holland, Amsterdam 